\title{
The Stem Cell-Expressed Receptor Lgr5 Possesses Canonical and Functionally Active Molecular Determinants Critical to $\beta$-arrestin-2 Recruitment
}

\author{
Joshua C. Snyder', Lauren K. Rochelle ${ }^{1}$, Larry S. Barak', Marc G. Caron ${ }^{1,2,3^{*}}$ \\ 1 Department of Cell Biology, Duke University Medical Center, Durham, North Carolina, United States of America, 2 Department of Neurobiology, Duke \\ University Medical Center, Durham, North Carolina, United States of America, 3 Department of Medicine, Duke University Medical Center, Durham, North \\ Carolina, United States of America
}

\begin{abstract}
Lgr5 is a membrane protein related to G protein-coupled receptors (GPCR)s whose expression identifies stem cells in multiple tissues and is strongly correlated with cancer. Despite the recent identification of endogenous ligands for Lgr5, its mode of signaling remains enigmatic. The ability to couple to $G$ proteins and ßarrestins are classical molecular behaviors of GPCRs that have yet to be observed for Lgr5. Therefore, the goal of this study was to determine if Lgr5 can engage a classical GPCR behavior and elucidate the molecular determinants of this process. Structural analysis of Lgr5 revealed several motifs consistent with its ability to recruit ßarr2. Among them, a "SSS" serine cluster located at amino acid position 873-875 within the C-terminal tail (C-tail), is in a region consistent with other GPCRs that bind ßarr2 with high-affinity. To test its functionality, a ligand-independent $\beta$ arr2 translocation assay was implemented. We show that Lgr5 recruits $\beta$ arr2 and that the "SSS" amino acids (873-875) are absolutely critical to this process. We also demonstrate that for full efficacy, this cluster requires other Lgr5 C-tail serines that were previously shown to be important for constitutive and ßarr2 independent internalization of Lgr5. These data are proof of principle that a classical GPCR behavior can be manifested by Lgr5. The existence of alternative ligands or missing effectors of Lgr5 that scaffold this classical GPCR behavior and the downstream signaling pathways engaged should be considered. Characterizing Lgr5 signaling will be invaluable for assessing its role in tissue maintenance, repair, and disease.
\end{abstract}

Citation: Snyder JC, Rochelle LK, Barak LS, Caron MG (2013) The Stem Cell-Expressed Receptor Lgr5 Possesses Canonical and Functionally Active Molecular Determinants Critical to $\beta$-arrestin-2 Recruitment. PLoS ONE 8(12): e84476. doi:10.1371/journal.pone.0084476

Editor: James Porter, University of North Dakota, United States of America

Received September 9, 2013; Accepted November 14, 2013; Published December 27, 2013

Copyright: @ 2013 Snyder, Rochelle. This is an open-access article distributed under the terms of the Creative Commons Attribution License, which permits unrestricted use, distribution, and reproduction in any medium, provided the original author and source are credited.

Funding: This work was supported by a Duke Cancer Center Stewart Trust and Duke Cancer Center Cancer and the Environment to JCS, LKR, LSB, and MGC; and NIDA P30 5P30DA29925 to LSB and MGC, and an NIMH F32 NRSA 5F32MH092013-03 to JCS. The funders had no role in study design, data collection and analysis, decision to publish, or preparation of the manuscript.

Competing interests: The authors have declared that no competing interests exist.

*E-mail: marc.caron@dm.duke.edu

\section{Introduction}

Lgr5 belongs to a family of $G$ protein-coupled receptors (GPCRs ${ }^{*}$ ) termed leucine-rich GPCRs (Lgr)s [1,2]. A subfamily of these receptors, include Lgr4-6, which possess large Nterminal extracellular domains that contain varying numbers of leucine-rich repeats (LRR)s. Lgr4-6 are classified as rhodopsinlike Class 1 GPCRs, because, despite their relatively oversized $\mathrm{N}$-termini, they also possess a seven transmembrane (7-TM) domain structure typical of GPCRs in general [3]. In the case of Lgr1-3 (FSHR, LHR, and TSHR, respectively), these N-terminal domains bind to heterodimeric cystine-knot protein hormones, and the receptors themselves couple to $G_{\alpha s}$ to stimulate cAMP production [4] and also engage ßarr2 [5-7].

In contrast to Lgr1-3, the biochemical and cellular details of signal transduction for Lgr4-6 are only beginning to emerge.
Since the discovery of Lgr4-6, their functions have remained elusive, due in part to an orphan status, meaning that functionally active cognate ligands remain unknown. In 2007, Barker et al demonstrated, by lineage tracing, that Lgr5 expression can be used to identify epithelial stem cells of the small and large intestine [8]. Since this discovery, Lgr5 mediated lineage tracing has reliably identified stem cells in several other tissues and interestingly Lgr5 expression has been correlated with cancer [9-11]. These findings fueled the search for cognate Lgr4-6 ligands and their signaling mechanism. In 2011 and 2012 several groups reported high affinity interactions of Lgr4-6 with Rspondins1-4 [12-16]. More recently, the cystine knot protein, Norrin, was also shown to interact with Lgr4 [17].

Incredibly, none of these high-affinity ligands seem able to induce classical GPCR behaviors such as coupling to one of 
the diverse G-proteins or engagement of the ßarrs. Ligand mediated activation of receptors and their subsequent phosphorylation by $\mathrm{G}$ protein-coupled receptor kinases (GRK)s stimulate the recruitment of $\beta$ arrs [18]. Once recruited, $\beta$ arrs regulate GPCR desensitization and endocytosis but also function as $G$ protein independent signaling scaffolds [19-23]. The inability to demonstrate that the high affinity Lgr4-6 ligands can direct either of these two GPCR behaviors has led some to even question whether Lgr4-6 are indeed GPCRs and therefore should be reclassified [10]. Thus, answering this fundamental question is of great importance in order to gain insight into the Lgr4-6 driven signaling program in stem cell and cancer cell behavior, as well as implementing drug discovery programs targeting this unique class of receptors.

To answer this question, we were aided by previous work in which the molecular determinants that mediate the constitutive internalization of Lgr5 to the trans-Golgi network were discovered. This unique property of Lgr5 was utilized in a series of structure and function studies, which revealed a serine motif in the $\mathrm{C}$-tail situated between amino acids 844 and 864 modulates this process. In particular, serine residues at position 861 and 864 were found to be critical to this phenomenon. A serine cluster "SSS" (aa873-875) downstream from this motif appears similar to those present in many other GPCRs that bind Barr2 and is a predicted GRK substrate, that surprisingly, wasn't necessary for Lgr5 internalization [24]. These data suggested that constitutive internalization occurs independent of $\beta$ arr2. However, whether this "SSS" cluster is a conserved $\beta$ arr2 recruitment motif that possesses any functional activity wasn't tested.

On this basis, we sought to determine if Lgr4-6, and in particular Lgr5, harbor protein motifs consistent with their ability to display classical GPCR behaviors. Lgr4-6 primary amino acid sequences were interrogated for the existence of molecular determinants conserved in other Class 1 GPCRs, which mediate classical GPCR behavior. We found that the aforementioned "SSS" cluster present in Lgr5 is a hypothetical high-affinity ßarr2 motif. Importantly, we demonstrate that Lgr5 can recruit ßarr2 in a GRK-dependent manner and that the "SSS" motif is fully functional and critical to this process. These data provide proof of principle that Lgr5 can engage classical GPCR behaviors. These results provide an important first step in assessing Lgr5 as a functional GPCR. Therefore, continued studies aimed at the discovery of Lgr5 orthosteric ligands and effectors supportive of classical GPCR behavior are warranted.

\section{Materials and Methods}

\section{Cloning and Plasmids}

Constructs encoding the Renilla reinformis green fluorescent protein (GFP) fused to the C-terminus of rattus norvegicus $\beta$ arrestin-2 ( $\beta$ arr2-GFP) or GFP fused to the C-terminus of $\beta$ arr1 (ßarr1-GFP) were previously described [25,26]. GRK2 (Bos taurus NM_174710), GRK4 (Homo sapiens NM_182982), GRK5 (Bos taurus NM_174331), and GRK6 (Homo sapiens NM_001004015) were available in the lab. The 3xHA-Nterminal epitope tagged human Lgr5 with an EGFP fused inframe at the C-terminus and phosphor-acceptor mutants were described previously [24]. Using these constructs, a stop sequence was inserted between the C-tail and EGFP fusion to eliminate EGFP expression and make these constructs amenable to the ßarr2-GFP translocation assay. Additional mutants not described in previous work were also generated using PCR mutagenesis, as previously described [24].

\section{Clustal Alignment}

The primary amino acid sequences of Human class 1 rhodopsin-like GPCRs were retrieved from the International Union of Basic and Clinical Pharmacology (IUPHAR) database [27] (http://www.iuphardb.org/DATABASE/GPCRListForward). Sequence alignments were generated using the Clustal $\Omega$ online web applet [28,29] (http://www.ebi.ac.uk/services/ proteins). Data were downloaded and uploaded into the freely available Jalview14.0 (http://www.jalview.org, downloaded and installed on Mac OSX, Cupertino, CA) for post analysis sequence visualization, sorting, and presentation [30]. Color coding of amino acid sequences was applied through ClustalX coloration and a consensus logo applied to calculate amino acid frequency for each conserved residue. Due to publishing constrains, only a subset of the receptors and their sequences in outlined in Figure 1. The complete data set is available as File S1 to be opened and viewed in Jalview. For analysis of Ctails only, full length sequences were downloaded from http:// www.uniprot.org in FASTA format, C-tail sequence isolated using the NPXXY motif as an identifier, uploaded and processed by Clustal $\Omega$, and visualized in Jalview 14.0, as described above.

\section{Barr2 Confocal Translocation Assays}

150,000 HEK 293 T/17 cells (HEK) (ATCC CRL-11268) cultured in DMEM (Mediatech/Cellgro 10-013-CV), 10\%FBS (Sigma F2242), and Antibiotic-Antimycotic (Invitrogen 15240-062) were plated in each well of 24-well glass bottom plates (MatTek Coporation, Ashton, MA (part\# P24G-0-10-F)) that were previously coated with with $75 \mu \mathrm{g} / \mathrm{ml}$ fibronectin in $\mathrm{H}_{2} \mathrm{O}$ (Sigma F2006). The next day cells were transfected with Barr2-GFP $(0.75 \mu \mathrm{g})$ plus the indicated receptor $(1 \mu \mathrm{g})$ and GRK $(1 \mu \mathrm{g})$ using a calcium phosphate transfection procedure [31] that was scaled back to $45 \mu \mathrm{I} \mathrm{H}_{2} \mathrm{O}, 5 \mu \mathrm{l} 2.5 \mathrm{M} \mathrm{CaCl}_{2}, 50 \mu \mathrm{l} 2 \mathrm{xHBS}$ per well. The next day cell media was changed to live cell staining media (SM) comprised of Clear MEM (Invitrogen 51200 ) plus 10mM HEPES (Invitrogen 15630) and 1xGlutaMAX-1 (Invitrogen 35050) or fixed with $4 \%$ paraformaldehyde for confocal imaging. Phosphor-acceptor mutants were blindly assessed for their ability to recruit ßarr2 over a minimum of three fields.

\section{Antibody Staining and Pulse-Chase Internalization}

Pulse-chase internalization assays were performed as previously reported [24]. Briefly, 350,000 HEK cells were plated on 12 well MatTek plates (MatTek Corporation, Ashton, MA P12G-0-10-F) that were coated with $75 \mu \mathrm{g} / \mathrm{ml}$ fibronectin in $\mathrm{H}_{2} \mathrm{O}$ (Sigma F2006). Cells were transfected the next day with indicated constructs using $0.75 \mu \mathrm{g}$ ßarr2-GFP, $1 \mu \mathrm{g}$ receptor, and $1 \mu \mathrm{g}$ GRK using the same protocol described above. The next day cells were washed once with ice cold SM and pulsed 


\section{Figure 1}

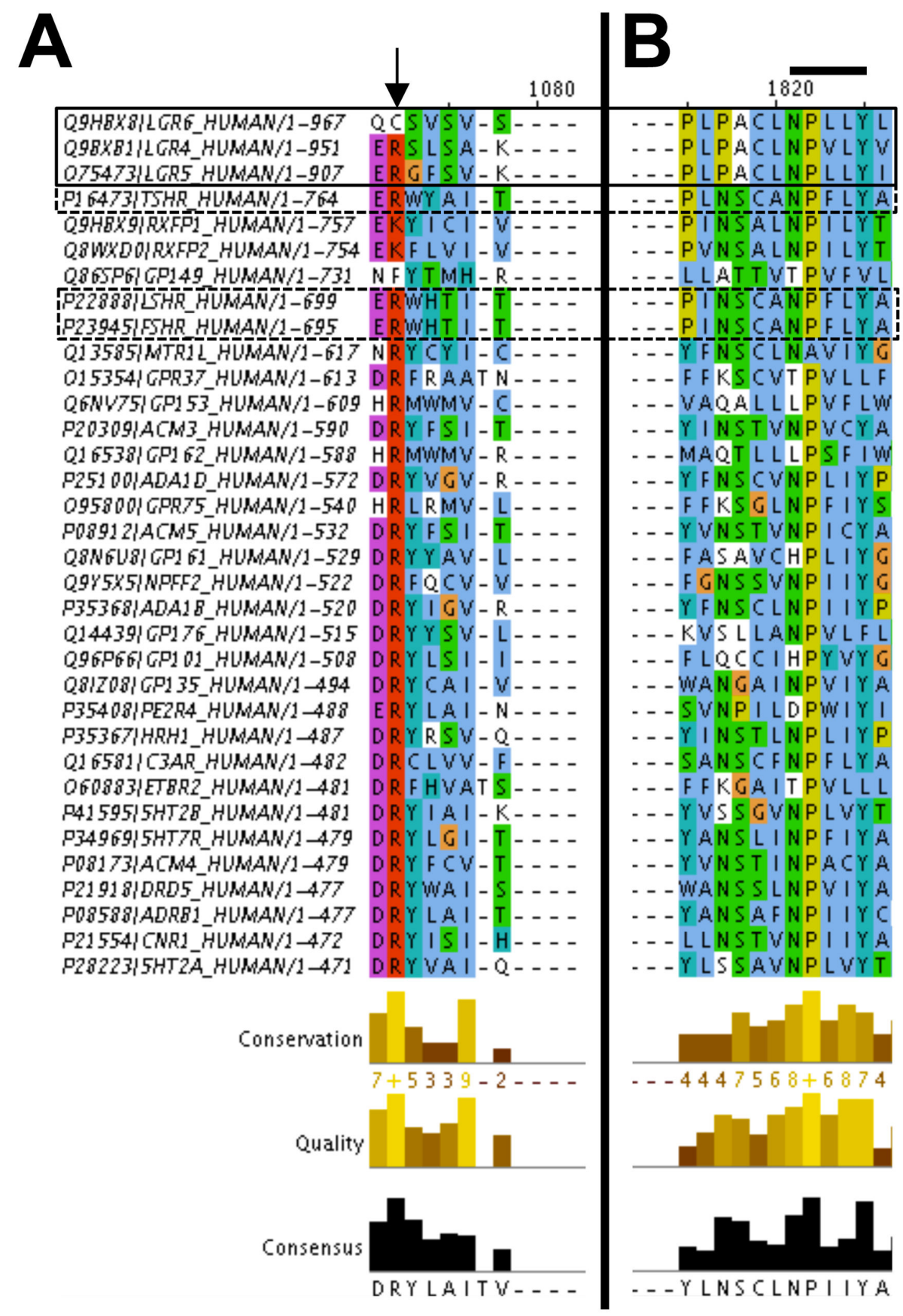

Figure 1. Conservation of typical GPCR signaling motifs in Lgr4-6. The primary amino acid sequences of 282 Class 1 rhodopsin-like GPCRs were aligned in Clustal $\Omega$ and a subset presented due to space limitations (ClustalX color view based upon degree of conservation; See jalview.org for complete color table, briefly, Acidic (E,D: Magenta); Basic (R, K:Red); (P: Yellow). The entire sequence alignment can be downloaded from File S1 and opened in Jalview. Lgr1-3 (FSHR, LSHR, and TSHR) and Lgr4-6 are highlighted by hatched and solid boxes. (A) Primary acid alignment from a subset of GPCRs at the "DRY" motif and (B) primary acid alignment from a subset of GPCRs at the NPXXY motif. Arrow in (A) points to the conserved Arginine in the "DRY" motif. Line in (B) highlights the NPXXY motif. The position label at top indicates amino acid position from the start of the alignment. At the bottom, alignment conservation, quality, and consensus for each amino acid (+, indicates significant conservation of the site). doi: 10.1371/journal.pone.0084476.g001 
with a MsaHA (from a mouse hybridoma available in the lab) at 1:500 in SM for 45 minutes, washed four times with SM, and fixed with $4 \%$ paraformaldehyde or chased for 2 hours in a $37^{\circ} \mathrm{C}$ cell culture incubator and then fixed similarly. Cells were permeabilized for ten minutes with $0.12 \%$ Triton-x-100/PBS and then blocked with $5 \% \mathrm{BSA} / 0.12 \%$ Triton- $\mathrm{x}-100$ for thirty minutes. Cells were then stained with GaM-568 (Invitrogen A11004) $1: 1000$ in blocking buffer for 1.5 hours. Cells were washed with PBS and then imaged by confocal microscopy. Antisera recognizing GRK5 and 6 (rabbit polyclonal made in house) or GRK6 specifically [Santa Cruz C20 (sc-566)] were kind gifts of Dr. Richard Premont (Figure S1).

\section{Confocal Analysis}

A Zeiss LSM 510 confocal microscope (Carl Zeiss Microimaging) equipped with appropriate laser lines and filter sets for $568 \mathrm{~nm}$, and $633 \mathrm{~nm}$ fluorescence imaging, was utilized to collect images. Images were acquired using a $100 \mathrm{X}$ objective and digital zoom set to 2x. A minimum of 3 images were collected for each sample.

\section{ArrestinZoom Assay}

35,000 HEK cells were plated onto a 96-well tissue culture plate (Thermoscientific 167008). A transfection master-mix was prepared by mixing $+/-1 \mu \mathrm{g}$ receptor, $0.75 \mu \mathrm{g}$ ßarr2-GFP, and $1 \mu \mathrm{g}$ GRK6 plasmid DNA in $90 \mu \mathrm{l}_{2} \mathrm{O}, 10 \mu \mathrm{l} 2.5 \mathrm{M} \mathrm{CaCl}$, and $100 \mu \mathrm{l} 2 x \mathrm{HBS}$, for a final volume of approximately $200 \mu \mathrm{l}$, enabled up to 8 technical replicate transfections $(25 \mu \mathrm{l} /$ well). Cells were transfected one day post plating and were cultured overnight. The next day, cells were fixed with $4 \%$ paraformaldehyde for ten minutes and then washed with PBS and stored at $4^{\circ} \mathrm{C}$. High-content large-field fluorescence imaging was performed using an Axiozoom.V16 (Carl Zeiss Microimaging). Barr2-GFP images were acquired with a GFPfilter set (Filter set $38 \mathrm{HE}$ : Ex BP 470/40, BS FT 495, Em BP $525 / 50)$ at $108 X$ magnification using the $2.3 x$ Lens. For each well of the 96 -well plate, 32 tiled-images (10\% overlap between images) were acquired using Zen 2012 Blue Edition (Carl Zeiss Microimaging). Using these parameters, $25 \%$ of the well surface was imaged for each well of a 96 well plate (totaling 3072 images or 1536 images, when 96 or 48 wells were respectively imaged). Imaging acquisition time was approximately 20 minutes for an entire 96 -well plate and only 10 minutes per 48-wells. A single stitched image was generated using the stitching function in Zen 2012 blue. For image processing, single high resolution non-compressed Tiff files were exported for each stitched well and imported into ImageJ [32]. Contrast and brightness were adjusted across the entire stitched image based upon the negative (ßarr2-GFP and GRK6) and positive control wells (Wild-type Lgr5, ßarr2-GFP, and GRK6). An image montage was created in ImageJ to reconstruct the 96-well plate format, a threshold was set to discriminate bright vesicular aggregates that formed in positive cells, and particles were analyzed and counted that had a minimum radius $>1.2$ pixels. Results were exported to Excel, and the number of vesicular aggregates totaled for a well in a Microsoft Excel pivot table. Bar graphs were generated in GraphPad Prism 5.0 (GraphPad Software) and statistically significant changes evaluated using a 1-way ANOVA and posthoc Bonferroni analysis $(p<0.05)$. A minimum of 4 technical replicates for each experimental condition were included in each of the four independently performed experiments.

\section{Results}

\section{Lgr4-6 possess classical GPCR structural motifs}

The structural requirements for coupling to $G$ proteins or $\beta$ arrs are contained within the receptor seven transmembrane domain (7-TM), intra-cellular loops, and the C-tail. Two of the most well-studied determinants include the DRY motif located in intracellular loop 2 (IC2) and the NPXXY motif immediately proximal to the C-tail at the end of transmembrane-7 (TM7) [33-37]. To probe for the existence of these motifs in Lgr4-6, Clustal $\Omega[28,29]$ analysis of the primary amino acid sequences of 282 human class 1 rhodopsin-like GPCRs was performed. Lgr1-3 (FSHR, LHR, and TSHR) and Lgr4-6 have substantial conservation of one or both of these motifs (Figure 1, hatched and solid boxes, respectively). In particular, Lgr4 and Lgr5 have substantial conservation of the critically important arginine (conserved in $93 \%$ of the receptors clustered) in the "DRY" motif (Figure 1A, arrow). A proline, nine amino acids downstream of the DRY motif, aids in high affinity ßarr2 binding. Consistent with previous reports, proline is conserved in $64 \%$ of rhodopsin-like GPCRs and conducive to a ßarr2 interaction (File S1) [35]. Lgr4 and Lgr5 instead have an isoleucine or serine at this position. Interestingly, a proline at this position isn't absolutely critical for ßarr2 coupling, as the $\alpha_{1 \mathrm{~B}}$-adrenergic receptor also harbors a serine and still interacts with Barr2 [38]. Lgr1-6 all have robust conservation of the "NPXXY" important for G protein coupling and engaging arrestin [34], including the consensus asparagine, proline and tyrosine, conserved in $70 \%, 93 \%$, and $83 \%$ of clustered receptors (Figure 1B, bold line). These results suggested that Lgr4-6 harbor bona fide structural determinants necessary for classical GPCR behaviors warranting a thorough search for additional motifs and characterization of their functional activity.

\section{The $\beta$ arr2 interaction motif found in the V2R is conserved in Lgr5 but not Lgr4 or Lgr6}

Upon ligand-mediated activation, GPCRs are phosphorylated by GRKs in the intracellular loops or in the C-tail to mediate the recruitment of $\beta$ arr2 $[18,19]$. The strength and duration of this interaction varies among receptors and includes the formation of receptor/arrestin complexes transiently at the cell membrane or a long-term interaction stable into receptor/arrestin intracellular endocytic vesicles. Receptors harboring distinct modes of $\beta$ arr2 recruitment have been classified as Class $A$ and Class B, respectively [38]. The motifs necessary for Class $B$ interactions are characterized by clusters of serine and threonine residues in the receptor tails downstream from the NPXXY motif and are substrates of GRKs [26,39,40]. Intriguingly a similar motif has already been characterized in the Lgr4-6 related human FSHR [5]. To more thoroughly determine if such functional motifs existed in Lgr4-6, Clustal $\Omega$ $[28,29]$ analysis of the $C$ - tails of these receptors alongside the Class A and Class B stereotypes, $\beta 2 A R$ and V2R, was 
performed (Figure 2). The V2R "SSS" and "TSS" motifs (Figure 2 , underlined) have been well studied, with the "SSS" absolutely critical for Class B ßarr2 recruitment [40]. Interestingly, only Lgr5 possesses a threonine and serine cluster, "TFTSSS" (Figure 2, underlined) that also aligns with a serine cluster in V2R. Previous work from our laboratory has demonstrated that activity of the serine/threonine clusters is dependent upon their distance from an evolutionarily conserved palmitoylated cysteine (Figure 2*) [41]. Lgr5 doesn't possess a conserved cysteine, however, the neighboring leucine is conserved (Figure 2, arrow) and when used as a landmark we found the "TFTSSS" motif to be present in approximately the correct location within the C-tail to be a putative ßarr2 recruitment determinant. Lastly, another interesting finding is the conservation of the "SCDS" motif in Lgr4-6 to the V2R and $\beta 2 A R$ in a similar locale (Figure 2, box), suggesting that this motif, which regulates internalization of Lgr5 may also possess either ßarr2 recruitment activity under certain stimuli or act as a priming site required for subsequent phosphorylation of the "TFTSSS" motif. From these data we set out to test the hypothesis that Lgr5 can engage ßarr2 through its conserved serine and threonine cluster motifs.

\section{GRK overexpression drives ligand independent recruitment of $\beta$ arr2 to Class $B$ receptors}

A ligand-independent assay was implemented to test this hypothesis, since Rspondins and Norrin are unable to promote ßarr2 translocation [12,17]. The ßarr2-GFP translocation assay, and its derivative technologies, have become robust, reliable, and facile assays for assessing GPCR activation [42-44]. On the basis that GRK overexpression can stimulate receptor phosphorylation and receptor sequestration, we sought to determine whether an assay for ligand-independent arrestin recruitment could be implemented using wild-type GRKs $[45,46]$. As a test for GRK-dependent ligand-independent arrestin recruitment, the prototypical Class $B$ receptor V2R (Figure 3, Left Panels) or the Class A B2AR (Figure 3, Right Pane/s) were used. Unstimulated V2R is unable to recruit GFPtagged $\beta$ arr2, which remains largely dispersed in the cytoplasm (Figure 3A). In contrast, overexpression of GRK2 or GRK4 in the presence of the V2R can cause translocation of ßarr2 to the plasma membrane in the absence of ligand (Figure 3B). Lastly, we found that GRK5 or GRK6 over-expression facilitated recruitment of $\beta$ arr2 to intracellular vesicles. In contrast, overexpression of GRK2/4/5/6 with the class A $\beta 2 A R$ was unable to initiate $\beta$ arr2 recruitment. These data indicate that this assay can feasibly probe for potential ßarr2 interactions, particularly those modulated by serine clusters within the C-tail of a receptor.

\section{GRK4-6 but not GRK2 overexpression can direct $\beta$ arr2 recruitment to Lgr5}

To test the effect of GRK on Lgr5, a Lgr5/V2R chimeric receptor generated previously was utilized, (Figure 4A-E, Middle Panels) in which the C-tail of Lgr5 was exchanged at amino acid 834 for the V2R C-tail from amino acids 343-371 [24]. In the absence of GRK overexpression, Barr2 remained dispersed in the cytosol (Figure 4A). However, upon overexpression of GRK2/4/5/6 (Figure 4B-E) we found a remarkable and robust recruitment of ßarr2 into intracellular vesicles, mirroring activation of a Class $B$ receptor. We repeated these experiments with wild-type Lgr5 to verify that this interaction could be recapitulated with its endogenous tail (Figure 4A-E, Right Panels). In both the absence of GRK overexpression or with GRK2 overexpression ßarr2 remained localized to the cytosol (Figure 4A,B). In contrast, overexpression of GRK4, GRK5, or GRK6 promoted ßarr2 translocation (Figure 4C-E). Interestingly, we observed a continuum of responses that included Class $A$ (membrane) to Class B (vesicular) ßarr2 translocation profiles. GRK4 appeared to be the weakest modulator and stimulated a Class A profile with only an occasional cell having appreciable ßarr2 vesicular localization. GRK5 possessed moderate activity in which cells with a mix of Class A and Class B Barr2-recruitment could be observed. Finally, GRK6 possessed the strongest activity in this assay and stimulated ßarr2 translocation to intracellular vesicles reminiscent of a Class $B$ response. As a control, the overexpression of GRKs only facilitates the translocation of Barr2 when receptors are also overexpresssed (Figure 4A-E, Left Panels).

In order to qualitatively assess the dependence of GRK overexpression on ßarr2 translocation in the ligandindependent assay, cells with endogenous GRKs or cells transiently transfected and overexpressing GRK5 or GRK6 plasmid were compared. The results confirm that the endogenous levels of GRKs are too low to support ligandindependent translocation of Barr2 to Lgr5 (Figure S1). Ligand mediated activation of GPCRs also promotes ßarr1 translocation to the activated receptor, and like ßarr2 translocation, it depends upon the molecular determinants in the receptor intracellular domains and C-tail [38]. In the ligandindependent system described, Barr1 translocation to Lgr5 or the V2R does not occur without or with overexpression of GRK2, GRK4, GRK5, or GRK6 (Figure S2). This suggests that Lgr5 either doesn't recruit ßarr1 or as for the V2R, ligandmediated activation is necessary for this interaction.

To verify that Barr2 and Lgr5 are interacting we co-stained for Lgr5 using an antibody directed toward a 3xHA-N-terminal epitope fused to Lgr5 (Figure 5). These data show that Lgr5 is unable to promote translocation of ßarr2 to the receptor with overexpressed GRK2, despite the high expression of Lgr5 and its localization to intracellular vesicles (Figure 5A,B), In contrast, GRK4 and GRK5 are able to facilitate ßarr2 translocation to Lgr5 punctae on the plasma membrane (Figure 5C,D). Remarkably, GRK6 overexpression causes ßarr2 translocation to large intracellular vesicles that contain Lgr5 (Figure 4E). Collectively, these data are proof-of-principle that structural determinants present in Lgr5 are functionally able to modulate classical GPCR behavioral processes, such as $\beta$ arr2 recruitment.

\section{ßarr2 recruitment to Lgr5 occurs during internalization and is dependent upon a motif within its C-terminal tail (834-907)}

ßarr2 binds to Class B receptors at the plasma membrane and traffics with the receptor during endocytosis into 


\section{Figure 2}

IQ9BXB1/LCR4_HUMAN/1-155 1075473/LCRS_HUMAN/1-92 (P30518)V2R_HUMAN/1-51 (P07550)/ADRB2_HUMAN/1-92 IQ9HBX8/LCR6_HUMAN/1-145

Conservation

Quality

Consensus

IQ9BXB1/LCR4_HUMAN/1-155 1075473/LCRS_HUMAN/1-92 (P30518)V2R_HUMAN/1-51 (P07550/ADRB2_HUMAN/1-92 IQ9HBX8/LCR6_HUMAN/1-145

Conservation

Quality

Consensus

IQ9BXB1/LCR4_HUMAN/1-155 1075473/LCRS_HUMAN/1-92 (P30518)V2R_HUMAN/1-51 (P07550)ADRB2_HUMAN/1-92 IQ9HBX8/LCR6_HUMAN/1-145

Conservation

Quality

Consensus

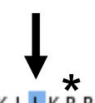

1 NPVLYVFFNPKFKEDWK LLKRRVTKKSGSVSV-SISSQGGCLEQDFY- - - - - - - YDCGMY- - - - S- 53 1 NPLLY I LFNPHFKEDLVSLRKQTYVWTR SKHPSLMSINSDDVEK- - - - - - - - - - - - - - - - - 1 NPWI YASFSSSVSSELRSLLCCAR - - - GRTPPSLG- - - PQD- - - - - - - - - - - - - 35 1 NPL I YCR - SPDFR IAFQELLCLRR SSLKA-YGNCY S SNGNTCEQSGYHVEQEKENKLLCEDLPGTEDFVG 68

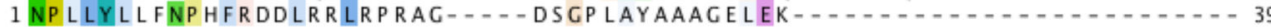

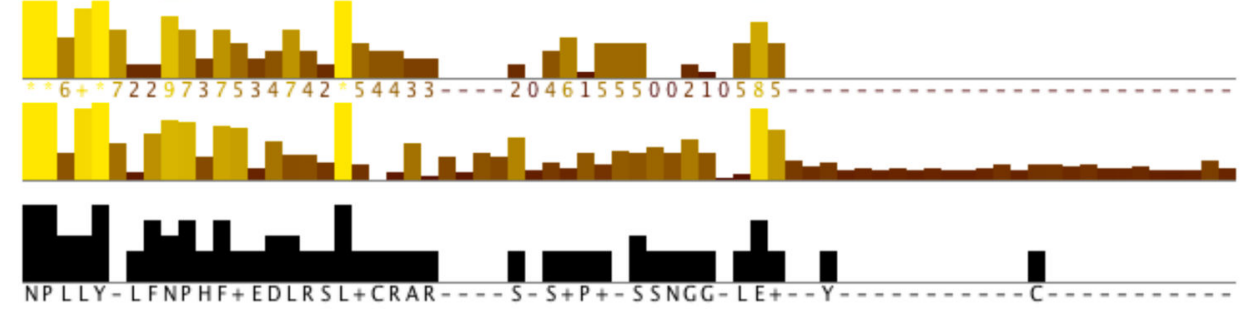

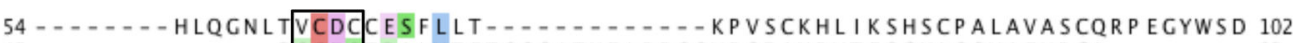

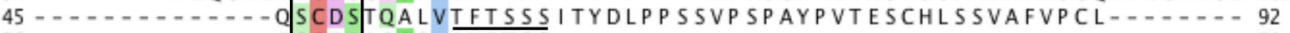
$36 \ldots \ldots \ldots \ldots$

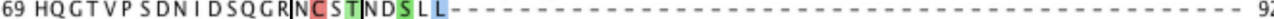
$40 \ldots$ SCDSTRALVAFSDVDLI LEASEAGRP . . PGLETYGFP SVTLISCQQPGAPRLE 91

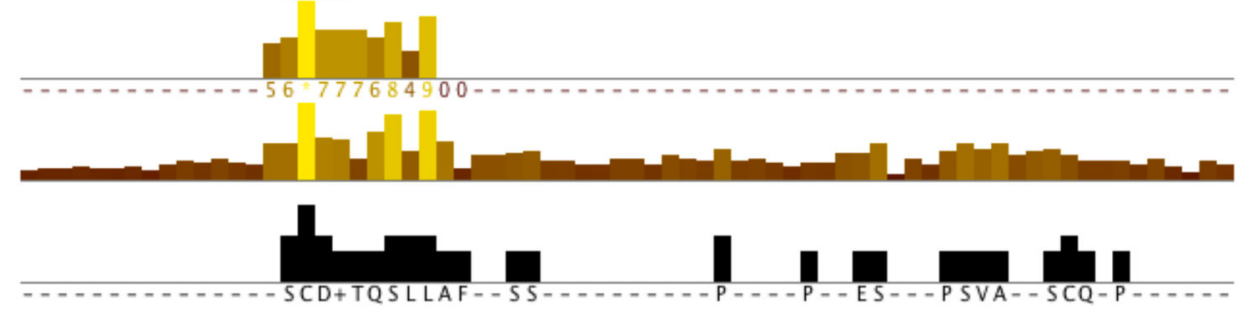

103 CCTQ - - SAHSDYADEEDSF-- - VSDSSDQVQACG- - - RACFYQSRGFPLVRYAYNLPRVKD (1) C-1-1- 92 GSHCVEPECNHFGNPQP SMDGELLLRAEGSTPAGGGLSGGGGFQP SGLAFASHV - - - . -
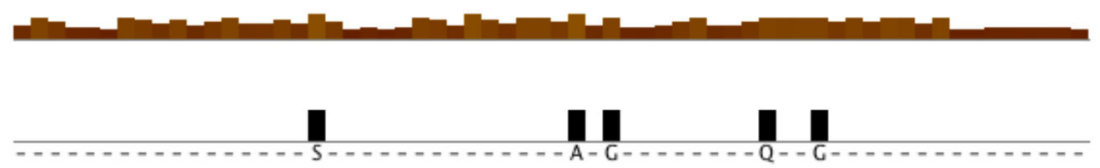

Figure 2. Conservation of a high-affinity interaction motif in Lgr5 but not Lgr4 or Lgr6. The C-terminal of tails of human Lgr4, Lgr5, Lgr6, V2R, and $\beta 2 A R$ (Uniprot IDs: Q9BXB1, O75473, Q9HBX8, P30518, and P07550) were aligned in Clustal $\Omega$ starting with the conserved NPXXY motif at the end of transmembrane seven (Clustal X color view based upon degree of conservation, See jalview.org for complete color table). Asterisk and arrow, respectively denote the position of the conserved palmitoylated cysteine (absent in Lgr4/5/6) and leucine. Underlined residues identify serine/threonine clusters which are necessary for Class B Barr2 affinity of the V2R. Of particular interest, is the conservation of a similar motif in Lgr5 (aa873-5) but not Lgr4, Lgr6, or $\beta 2 A R$. The boxed residues represent conservation of the motif necessary for Lgr5 internalization that are also seen in the V2R and $\beta 2 A R$. doi: 10.1371/journal.pone.0084476.g002

intracellular vesicles [40]. Lgr5 is rapidly internalized to the trans-Golgi network (TGN) following its delivery to the cell surface [24]. Therefore, we sought to determine if the translocation of ßarr2 to Lgr5 vesicles occurred with internalized or with de novo synthesized receptors. Lgr5 (Nterminally 3xHA Epitope tagged) and GFP tagged ßarr2 were 


\section{Figure 3}
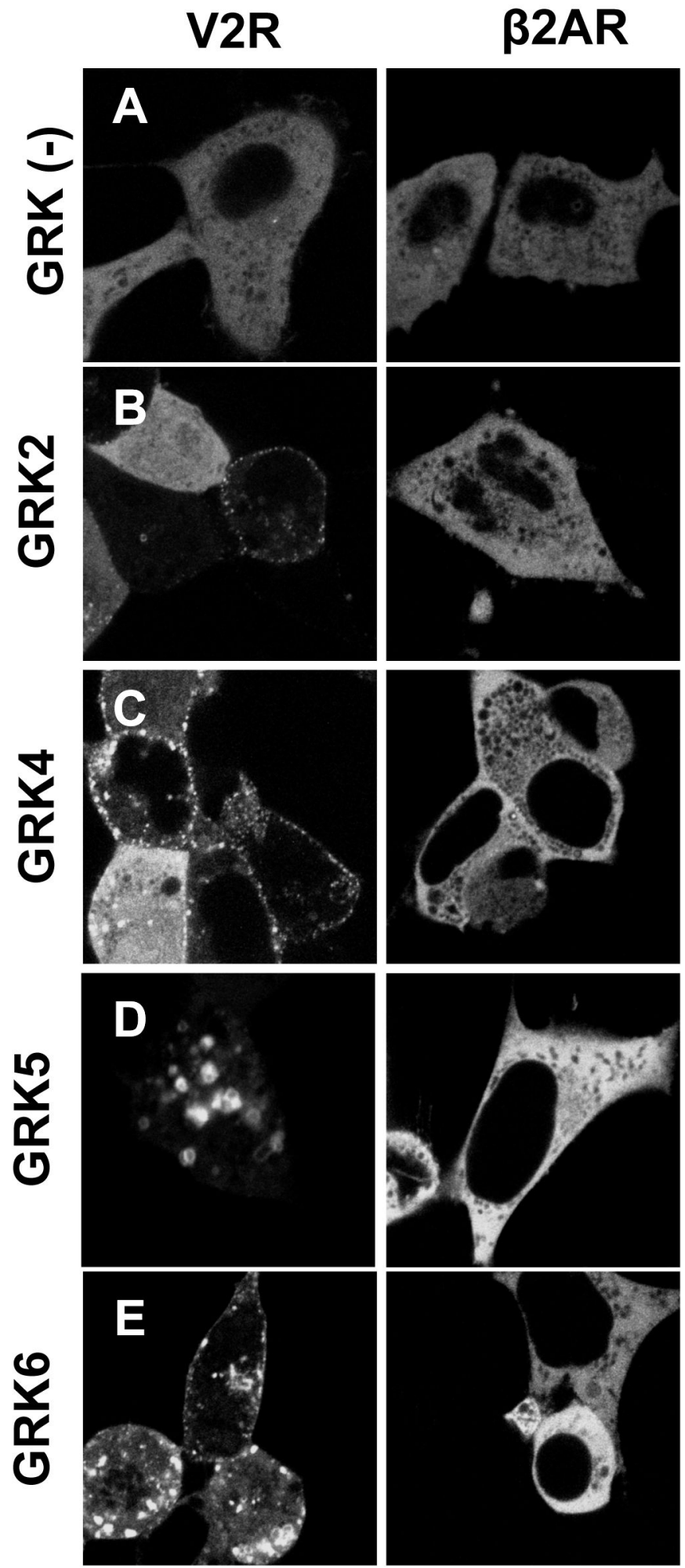

Figure 3. GRK overexpression drives ligand independent $\beta$ arr2 translocation to Class $B$ but not Class A GPCRs. Typical Class B (V2R, left) or Class A (B2AR, right) GPCRs were transiently co-expressed with GFP tagged ßarr2 in HEK 293 cells in (A) the absence of GRK overexpression or in the presence of overexpressed (B) GRK2, (C) GRK4, (D) GRK5, or (E) GRK6. Native GFP fluorescence was imaged at 100X with a confocal microscope and representative images presented.

doi: 10.1371/journal.pone.0084476.g003 


\section{Figure 4}

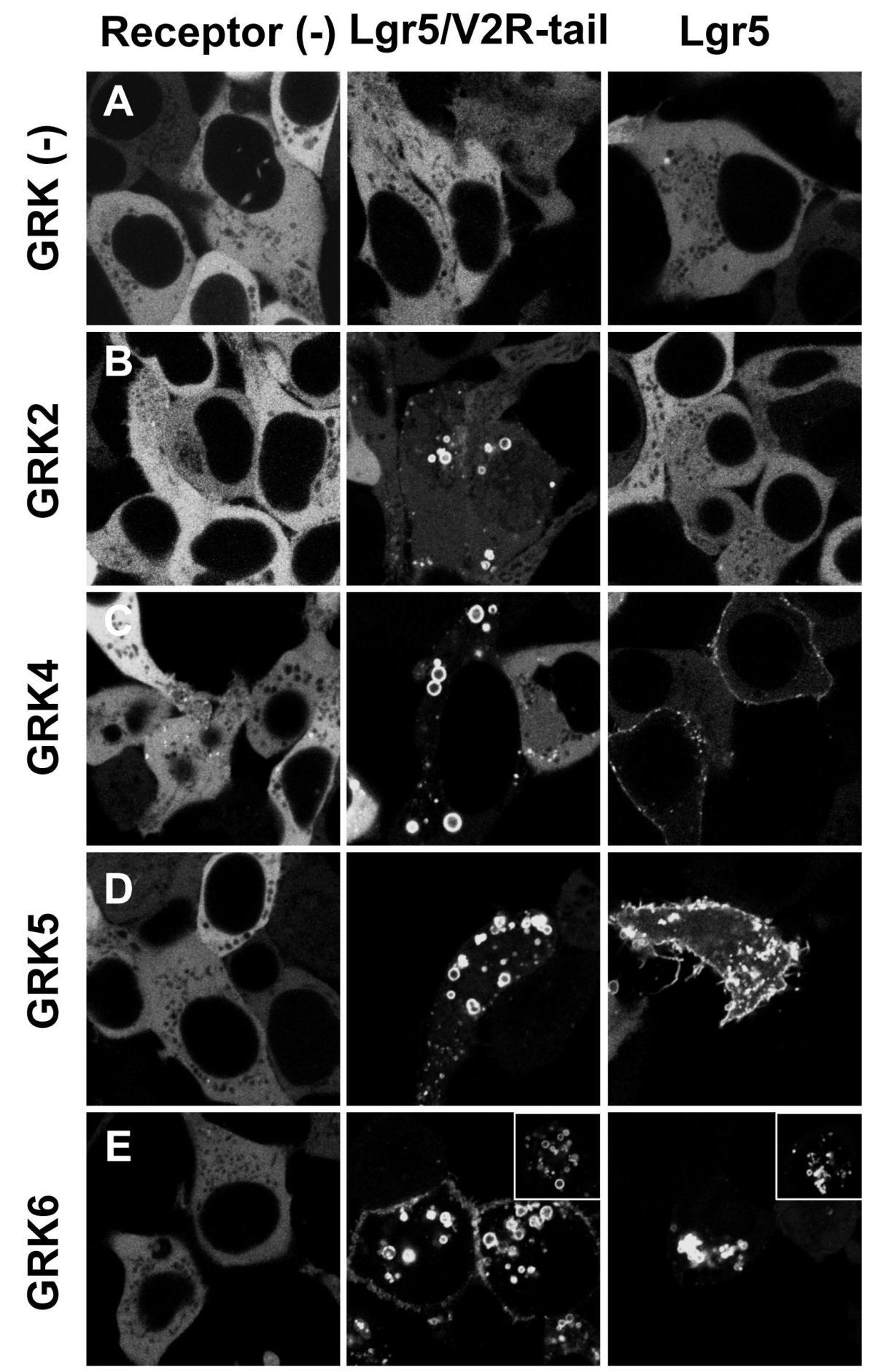

Figure 4. GRK overexpression mediates $\beta$ arr2 translocation to Lgr5. GFP tagged $\beta$ arr2 was transiently transfected in HEK 293 cells alone (Left Panels) or in the presence of overexpressed Lgr5/V2R chimera (Middle Panels) or wild-type HA epitope-tagged Lgr5 (Right Panels). Barr2-GFP translocation was visualized using a confocal microscope at 100X in (A) the absence of overexpressed GRK2 or in the presence of overexpressed (B) GRK2, (C) GRK4, (D) GRK5, or (E) GRK6. Inset depicts 2x magnification of images.

doi: 10.1371/journal.pone.0084476.g004 


\section{Figure 5}
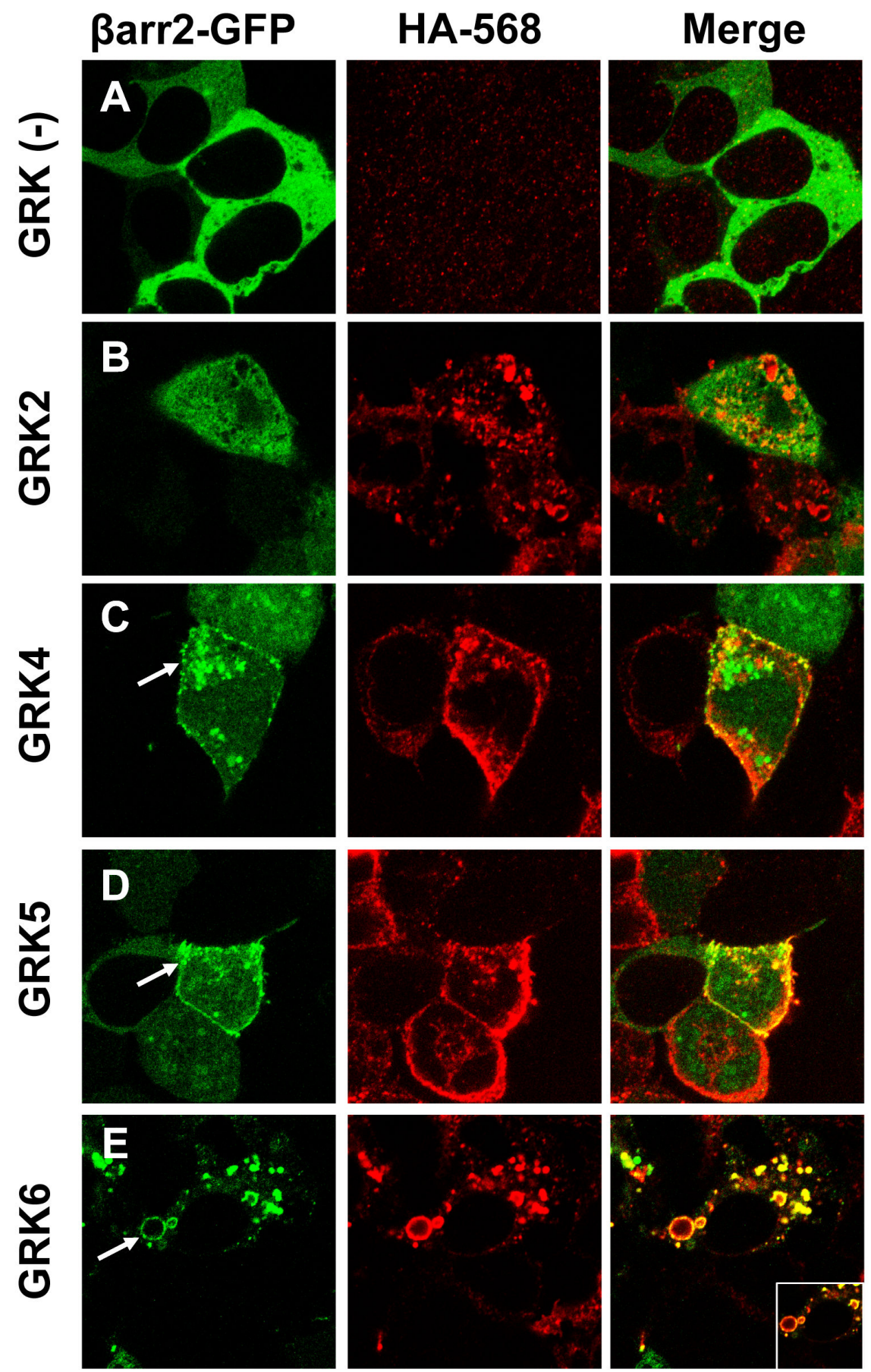

Figure 5. GRK6 overexpression mediates $\beta$ arr2 recruitment to Lgr5 intracellular vesicles. GFP-tagged $\beta$ arr2 and HA epitope-tagged Lgr5 was transiently overexpressed in HEK cells (A) without GRK overexpression, or with overexpression of (B) GRK2, (C) GRK4, (D) GRK5, or (E) GRK6. Cells were fixed, permeabilized, and stained with a primary and secondary (568nm) antibody pair to HA. Representative images are presented of native GFP fluorescence (Left Panels, Green), HA-568 (Middle Panels, Red), and merged (Right Panels, yellow denotes colocalization). Arrows point to representative $\beta$ arr2/Lgr5 colocalization. Inset depicts a $2 x$ magnification.

doi: 10.1371/journal.pone.0084476.g005 
transfected and cells were pulsed with primary antibody, washed, and chased for 0 or 120 minutes to stain surface expressed receptor and follow its trafficking. For wild-type Lgr5 (Figure 6, A-D, Left Panels), as expected, we found that GRK2 overexpression didn't promote Barr2 translocation and colocalization with Lgr5 at the membrane at time 0 minutes nor following Lgr5 trafficking to intracellular vesicles after 120 minutes of chase (Figure 6A). As we have already shown, overexpression of either GRK4 or GRK5 caused translocation of Barr2 to the membrane (Figure 6B,C, $0 \mathrm{~min}$ ). Importantly, this interaction is only transient, as few Barr2/Lgr5 positive vesicles were identified when Lgr5 was allowed to internalize for 120 minutes (Figure 6B,C, $120 \mathrm{~min}$ ). In contrast, in the setting of GRK6 overexpression, a remarkable colocalization of Lgr5 and ßarr2 in endocytic vesicles 120 minutes into the chase was found (Figure 6D).

As further evidence that the principal determinant for this interaction is a component in the C-tail of Lgr5 we also truncated the C-terminus at amino-acid position 834 (834del). In contrast to wild-type Lgr5 the internalization of 834del Lgr5 is largely blunted [24]. Moreover, even in the presence of GRK2/4/5/6, Barr2 fails to translocate (Figure 6A-D respectively). These data indicate that the Lgr5- $\beta$ arr2 interaction is driven by a protein motif residing in the distal Ctail.

\section{The "SSS" motif (aa873-875) in Lgr5 is necessary for recruitment of $\beta$ arr2}

To determine if the threonine and serine cluster in the C-tail acts as a typical GPCR ßarr2 translocation motif in Lgr5, ßarr2 translocation assays with $\mathrm{C}$-tail truncation mutants were performed. For these studies, we focused on GRK6 driven recruitment to Lgr5 (Figure 7A). Truncations of Lgr5 at amino acid position 864 and 875 were used, based upon the proximity of these residues to Lgr5 internalization motifs and putative Barr2 interacting motifs (Figure 6B). Compared to the truncation at 834 , restoring the C-tail to position 864 didn't facilitate the translocation of Barr2 (Fig. 7B.1, 2, Table 1). In contrast, truncation of Lgr5 at amino acid position 875 resulted in a receptor still capable of recruiting Barr2 (Fig. 7B.3, Table 1).

These data provide evidence that an amino acid motif between 865 and 875 is necessary for GRK6 dependent translocation of Barr2 to Lgr5. Near this region is serine 864 , previously shown to regulate constitutive internalization of Lgr5. Mutating this serine alone to alanine had no effect (Figure $7 B$. 4, Table 1), as would be expected based upon Fig. 7B.2. To determine if phosphorylation could play a role, all potential phosphor-acceptors between amino acid residue 866 and 907 were mutated (pDel 866-907). Results demonstrated that this construct was unable to support ßarr2 translocation (Fig.7B.5, Table 1). The serine/threonine cluster is present within this region (amino acid position 873-875). Therefore, this serine cluster was mutated to alanine. Remarkably, this receptor was also unable to recruit $\beta$ arr2. In contrast to Lgr5, Lgr4 doesn't possess this "SSS" motif and as expected doesn't support GRK mediated ßarr2 translocation (Figure 7C). These data demonstrate that that the "SSS" at position $873-875$ is required for Barr2 translocation to Lgr5.

\section{Cooperation between the internalization motif "SCDS" and the "SSS" cluster drives GRK6 dependent $\beta$ arr2 recruitment to Lgr5}

To determine if a motif other than the "SSS" was required for ßarr2 recruitment we performed an unbiased screen of all phosphor-acceptors in the C-tail. Alanine substitution mutants were generated at potential phosphorylation sites (Table 1) and were characterized by their ability to recruit Barr2 in a GRK6 dependent manner (Fig. 8B.1-12) relative to wild-type Lgr5 (Figure 8A). As expected, mutation of all potential phosphorylation sites from amino acid 833 to 907 (pDEL 833-907) inhibited ßarr2 recruitment (Fig. 8B.1). Previously, we have shown that the constitutive internalization of Lgr5 occurs independent of the "SSS" cluster (aa873-875) and ßarr2 but rather upon the serine pair 861/864. Therefore, we tested whether these residues or upstream serines were potential priming sites necessary for the functionality of the "SSS" motif. Potential phosphorylation sites from amino acid positions 833-865 (pDEL 833-865) or 844-864 (pDEL 844-864) were mutated to alanine (Table 1). Neither receptor mutant was able to cause translocation of Barr2 (Fig. 8B.2,3). To identify the phosphorylation sites necessary within 844-864, each site was systematically returned to its wild-type residue using the pDEL 844-864 construct as a template (Table 1). In these experiments, +A844S, +A848S, and +A851S, restored Barr2 translocation activity, while $+A 854 S$ only weakly restored this activity (Figure 8B.4-7). These residues were previously shown to be dispensable for internalization, so next we focused on the serine pair at amino acid position 861/864 which robustly affects Lgr5 internalization. Restoration of these residues in tandem (Table 1, Figure $8 B .8$ or singularly restored $\beta$ arr2 translocation (Table 1, Figure $8 B .9,10$ ), though restoration at serine 861 didn't restore the Class B activity. Finally, the necessity of these residues was tested by mutating only these residues in tandem and singularly in an otherwise wild-type receptor. Tandem mutation of $861 / 4$ to alanine markedly reduced $\beta$ arr2 translocation, eliminating Class $B$-like vesicular translocation and yet preserving some weak membrane Class A-like activity (Figure $8 B .11$ and Table 1). In contrast, selective mutation, of either serine 861 or 864 , to alanine failed to perturb $\beta$ arr2 translocation (Figure $8 B .12$ and Figure 7B.4 and Table 1). Collectively, these data indicate that the serine motifs present within the internalization motif of Lgr5 (aa844-864) and the "SSS" cluster (aa873-5) act cooperatively to facilitate GRK mediated Barr2 translocation to Lgr5.

\section{Quantitative analysis of Barr2 translocation to Lgr5 using wide-field high resolution imaging}

To assess ßarr2 translocation to Lgr5 in an unbiased manner, a robust and reliable methodology to quantify ßarr2GFP translocation was developed, which we refer to as ArrestinZoom. As opposed to present high-content, medium to high throughput imaging strategies, the ArrestinZoom assay utilizes a relatively inexpensive microscopy platform. This system is capable of providing both low and high resolution 


\section{Figure 6}

\section{Lgr5}
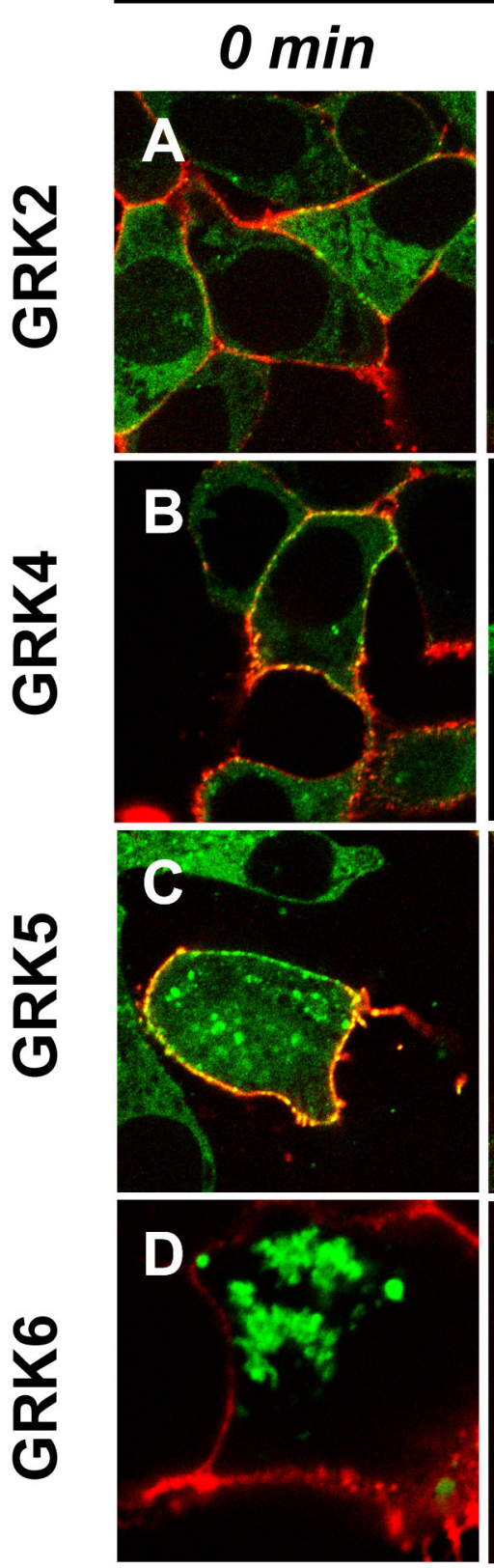

\section{$120 \min$}
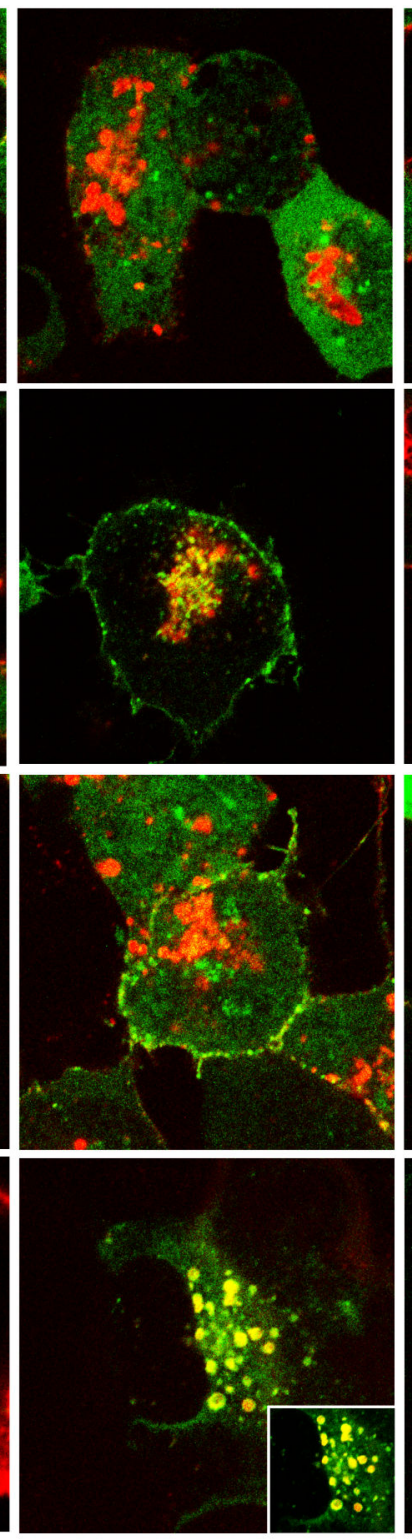

834del
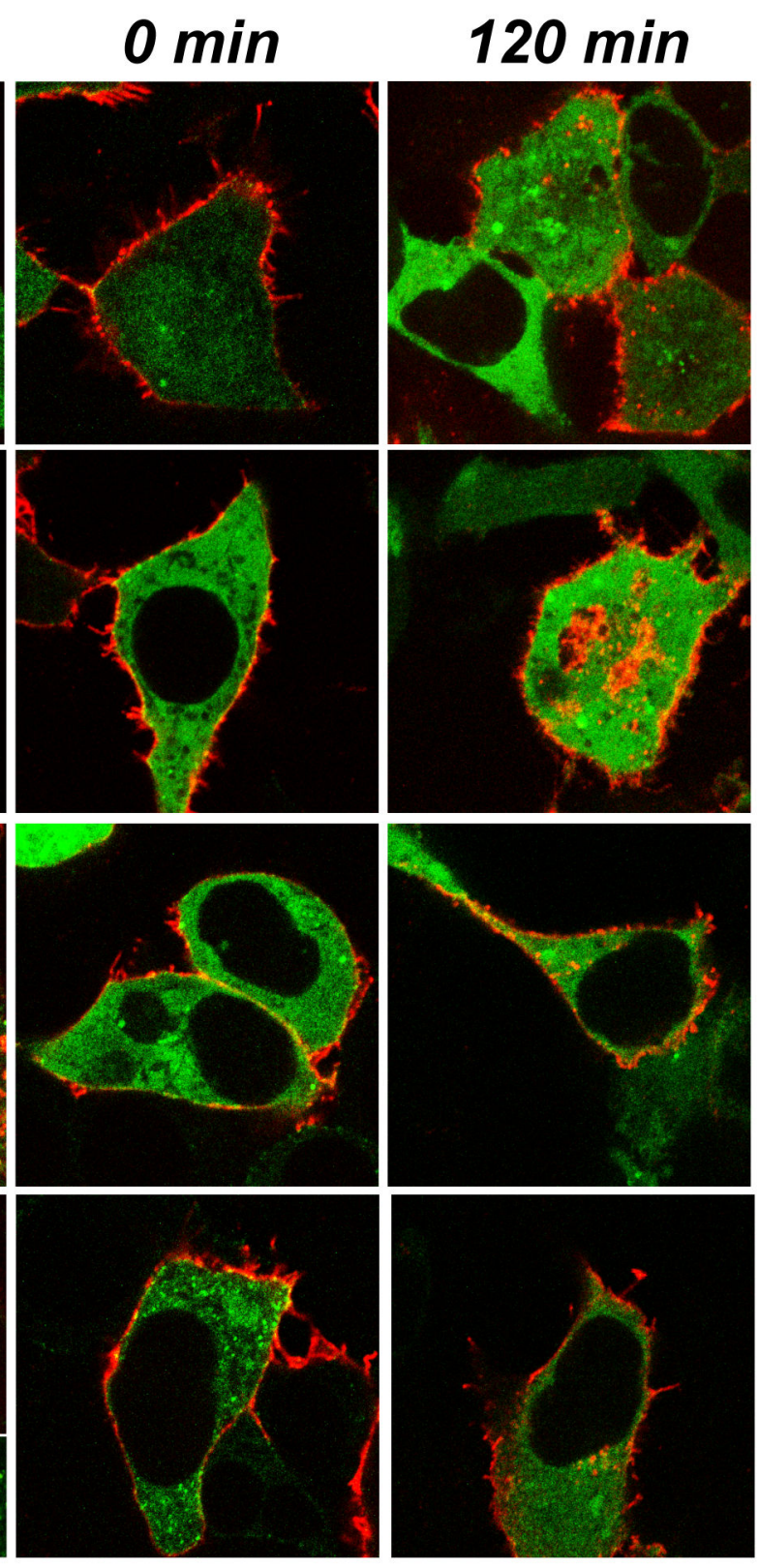

Figure 6. GRK6 overexpression stimulates Class B ßarr2 translocation to Lgr5 that is dependent upon a structural determinant in the C-tail. (Left panels) Wild-type HA epitope-tagged Lgr5 or (Right panels) HA epitope-tagged 834del (See Table 1 for truncation site) Lgr5 were cotransfected in HEK293 cells with GFP-tagged ßarr2 and (A) GRK2, (B) GRK4, (C) GRK5, or (D) GRK6. Cells were pulsed with an HA antibody on ice, washed, chased, and fixed. Cells were permeabilized and stained with a secondary antibody to the primary HA antibody. Images were collected on a confocal microscope at 100X. (Red:HA and Green:Native GFP were Merged:yellow). Inset depicts 2x magnification.

doi: 10.1371/journal.pone.0084476.g006

wide-field and higher-magnification images using a high numerical aperture zoom lens. When coupled to a low noise
CCD camera, motorized stage, and appropriate image acquisition software we were able to acquire thousands of 
Table 1. Overview of constructs utilized in Figures 7 and Figure 8.

\begin{tabular}{|c|c|c|}
\hline \multirow[t]{2}{*}{ Fig. } & Receptor & Primary Amino Acid Sequence \\
\hline & Wild-type & SLRKQTYVWTRSKHPSLMS INSDDVEKOSCDSTQALVTFTSSS ITYDLPPSSVPSPAYPVTESCHLSSVAFVPCL \\
\hline 7.1 & $834 \mathrm{del}$ & SL \\
\hline 7.2 & $864 \mathrm{del}$ & SLRKQTYVWTRSKHP SLMS INSDDVEKQSCDS \\
\hline 7.3 & $875 \mathrm{del}$ & SLRKQTYVWTRSKHPSLMS INSDDVEKQSCDSTQALVTFTSSS \\
\hline 7.4 & S864A & SLRKQTYVWTRSKHP SLMS INSDDVEKQSCDATQALVTFTSSS I TYDLPPSSVP SPAYPVTESCHLSSVAFVPCL \\
\hline 7.5 & pDEL 866-907 & SLRKQTYVWTRSKHP SLMS INSDDVEKQSCD STQALVAFAAAA IAADLP PAAVPAPAAPVAEACHLAAVAFVPCL \\
\hline 7.6 & S873-5A & SLRKQTYVWTRSKHP SLMS INSDDVEKQSCD STQALVTFTAAAI TYDLPP SSVP SPAYPVTESCHLSSVAFVPCL \\
\hline 8.1 & pDEL 833-907 & ALRKQAA VWARAKHPALMA INADDVEKQACDAAQALVAFAAAA IAADLP P AAVPAPAAPVAEACHLAAVAFVPCL \\
\hline 8.2 & pDEL 833-865 & ALRKQAAVWARAKHPALMAINADDVEKQACDAAQALVTFTSSS I TYDLPP SSVP SPAYPVTESCHLSSVAFVPCL \\
\hline 8.3 & pDEL 844-864 & SLRKQTYVWTRAKHPALMAINADDVEKQACDATQALVTFTSSS I TYDLPP SSVP SPAYPVTESCHLSSVAFVPCL \\
\hline 8.4 & pDEL $844-864+A 844 S$ & SLRKQTYVWTRSKHPALMAINADDVEKQACDATQALVTFTSSS I TYDLPP SSVP SPAYPVTESCHLSSVAFVPCL \\
\hline 8.5 & pDEL $844-864+A 848 S$ & SLRKQTYVWTRAKHP SLMAINADDVEKQACDATQALVTFTSSS I TYDLPP SSVP SPAYPVTESCHLSSVAFVPCL \\
\hline 8.6 & pDEL $844-864+A 851 S$ & SLRKQTYVWTRAKHPALMSINADDVEKQACDATQALVTFTSSS I TYDLPP SSVP SPAYPVTESCHLSSVAFVPCL \\
\hline 8.7 & PDEL $844-864+A 854 S$ & SLRKQTYVWTRAKHPALMAINSDDVEKQACDATQALVTFTSSS ITYDLPPSSVP SPAYPVTESCHLSSVAFVPCL \\
\hline 8.8 & pDEL $844-864+A 861 / 4 S$ & SLRKQTYVWTRAKHPALMAINADDVEKQSCD STQALVTFTSSS I TYDLPPSSVP SPAYPVTESCHLSSVAFVPCL \\
\hline 8.9 & PDEL $844-864+A 861 S$ & SLRKQTYVWTRAKHPALMAINADDVEKQSCDATQALVTFTSSS I TYDLPP SSVP SPAYPVTESCHLSSVAFVPCL \\
\hline 8.10 & pDEL $844-864+A 864 S$ & SLRKQTYVWTRAKHPALMAINADDVEKQACD STQALVTFTSSS I TYDLPPSSVP SPAYPVTESCHLSSVAFVPCL \\
\hline 8.11 & $\mathrm{~S} 861 / 4 \mathrm{~A}$ & SLRKQTYVWTRSKHP SLMS INSDDVEKQACDATQALVTFTSSS I TYDLPPSSVP SPAYPVTESCHLSSVAFVPCL \\
\hline 8.12 & S861A & SLRKQTYVWTRSKHP SLMS INSDDVEKQACD STQALVTFTSSS I TYDLPP SSVP SPAYPVTESCHLSSVAFVPCL \\
\hline
\end{tabular}

Constructs were aligned at amino acid sequence 833. In bold are serine, threonine, or tyrosine residues that were mutated to alanine (A). Serines that were returned to wildtype sequence are shown as italicized bold $(S)$.

doi: 10.1371/journal.pone.0084476.t001

images from a 96-well plate (32images/well = 3072images). The ArrestinZoom assay was applied to all of the Lgr5 constructs previously tested in the confocal translocation assay as discussed in materials in methods. Briefly, multiple tiled images from each well were stitched and then processed in ImageJ to identify ßarr2 aggregates. Results of this analysis demonstrated visually that the formation of $\beta$ arr2 aggregates was dependent upon Lgr5 expression (Figure 9A and 10A) and that the $\mathrm{C}$-tail is critical to this process. Importantly, as the complementary confocal translocation analysis demonstrated, the serine cluster "SSS" from amino acid 873-875 (Figure 9A; Compare Box 3 (S873-5A) to Box 2 (WT)) is necessary for Lgr5-dependent ßarr2 translocation and the upstream phosphor-acceptors also play a cooperative role in this process (Figure 10A; Compare Box 3 (pDEL 844-864) to Box 2 (WT)). The boxes outlined in Figure $9 \mathrm{~A}$ and $10 \mathrm{~A}$ are also presented at 8.9X (Figure 9B and 10B) and 108X (Figure 9C and 10C) magnification to demonstrate at the cellular level the robust formation of Barr2 aggregates for WT Lgr5 (Box 2, arrow) and their absence in the negative control (Box 1) or phosphoracceptor mutant (Box 3). Lastly, the identified Barr2 aggregates in $9 \mathrm{~A}$ and $10 \mathrm{~A}$ were counted and graphically presented (Figure 9D and 10D). These results demonstrated quantitative and statistical differences in $\beta$ arr2 aggregate formation for Lgr5 and its mutant derivatives that confirmed the confocal imaging experiments. The results of this study demonstrate for the first time that Lgr5 can recruit ßarr2 and begin to identify the molecular determinants of this process.

\section{Discussion}

The signaling pathways potentially engaged by the proposed high-affinity ligands to Lgr4-6 remain enigmatic. Rspondins facilitate Lgr4-6 mediated Wnt-signaling while Norrin only stimulates Lgr4 mediated Wnt-signaling, despite high affinity binding to Lgr5 and 6 [12-17]. Interestingly, each ligand shares a curious inability to engage typical GPCR behavior, such as G protein-coupling or $\beta$ arr2 recruitment. These observations have caused some to question whether Lgr4-6 are even GPCRs: "Surprisingly, G-protein signaling does not seem to be involved in mediating Lgr5-homolog- derived Wnt signals, raising the question of whether their classification as GPCR's is accurate [10]."

To fully elucidate the impact that Lgr4-6 signaling plays in cellular behavior, mandates that this question be answered. Therefore, in this study we sought to determine whether Lgr4-6, in particular Lgr5, are able to support classical GPCR behaviors. We demonstrated that Lgr4-6 harbor typical GPCR structural motifs and that Lgr5, in particular, possesses a serine cluster reminiscent of those present in GPCRs capable of high affinity ligand-mediated Barr2 recruitment. Our recent work defined the principal motifs regulating Lgr5 internalization and trafficking to the TGN. Importantly, we demonstrated previously that aa873-875 ("SSS") are dispensable for internalization [24]. In this study, we show that Lgr5 can recruit Barr2 and that the "SSS" residues are absolutely necessary for this particular process. An unanticipated finding was that $\beta$ arr2 translocation to Lgr5 also required upstream serines from amino acid 844-864, which together were shown to be essential for the 


\section{Figure 7}
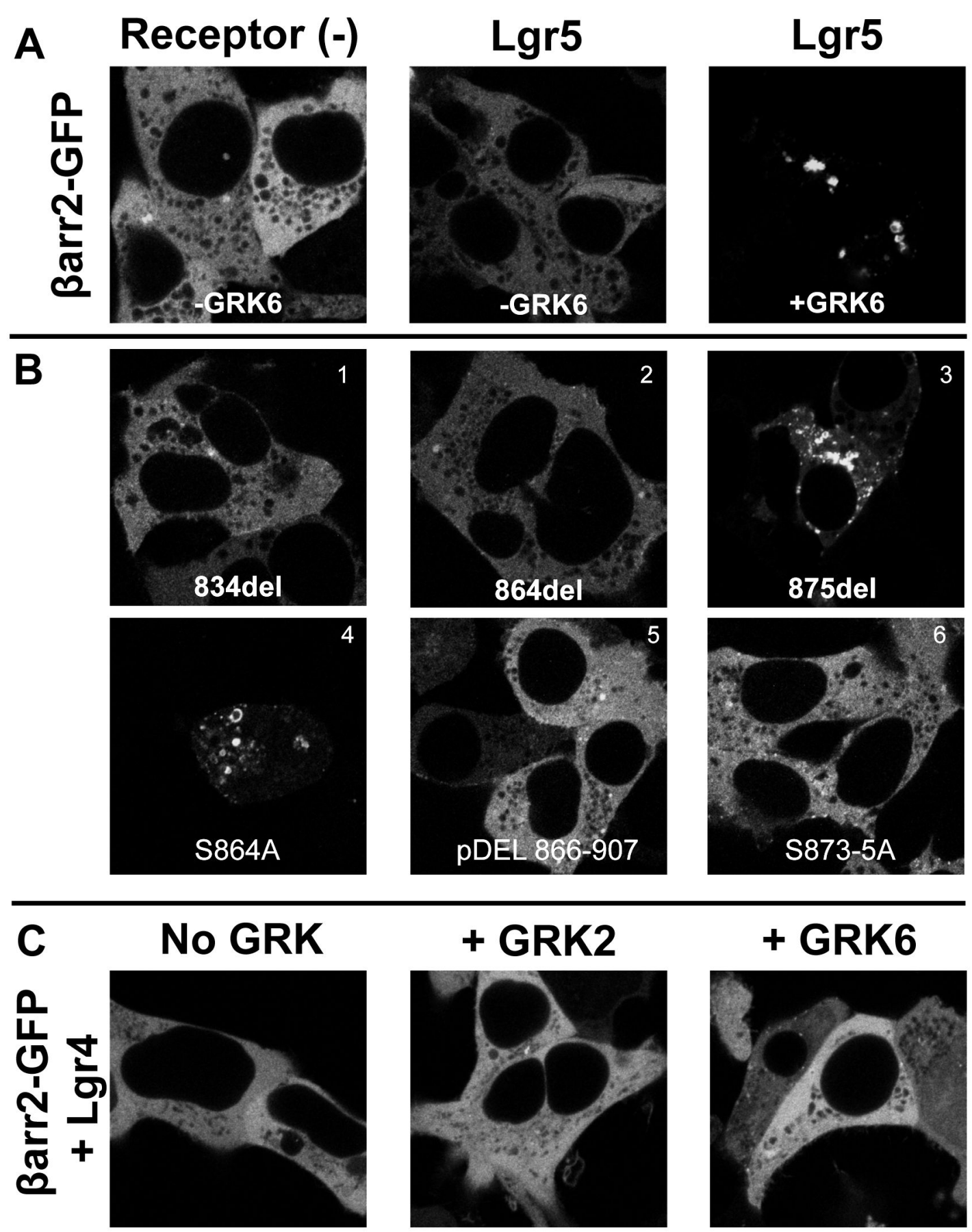

+ GRK2

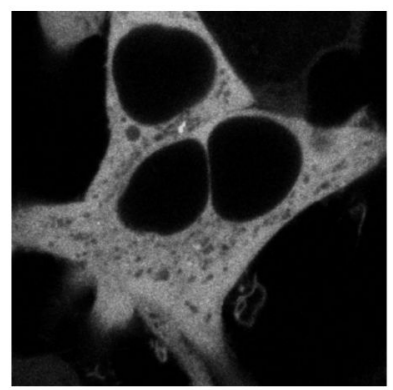

+ GRK6

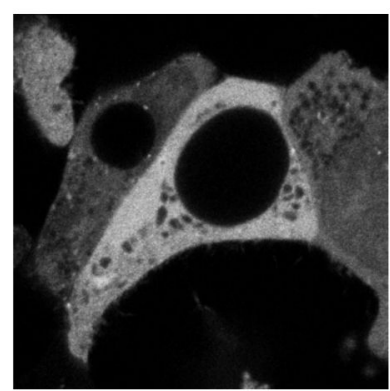

Figure 7. The amino acids "SSS" from position 873-875 in Lgr5 mediate ßarr2 translocation. (A) GFP-tagged ßarr2 was transiently expressed alone (Left panel) or with wild-type Lgr5/-GRK6 (Middle panel) or Lgr5/+GRK6 (Right panel) in HEK 293 cells. (B) GFP-tagged Barr2 and GRK6 were transiently expressed with the indicated C-tail mutants. The primary acid sequence of the Ctail mutants is provided in tabular form (Table 1 7.1-.6). (C) GFP-tagged Barr2 and Lgr4 were transiently expressed in HEK293 cells in the absence of GRK overexpression or with GRK2 or GRK6 (Left, Middle, and Right panels, respectively). Native GFP fluorescence was imaged by confocal at $100 \mathrm{X}$.

doi: 10.1371/journal.pone.0084476.g007 


\section{Figure 8}
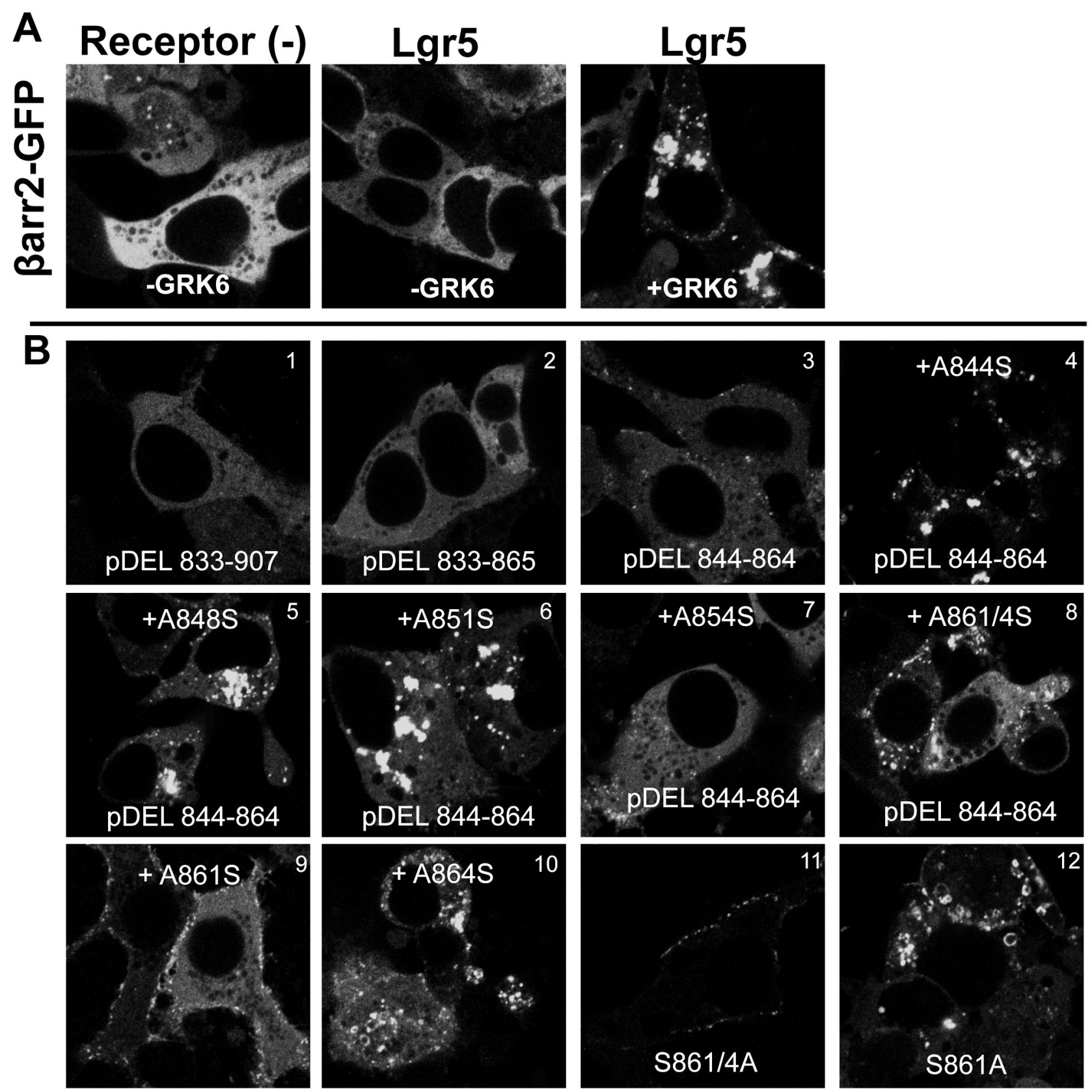

Figure 8. Cooperation of Lgr5 internalization motifs is necessary for "SSS" mediated ßarr2 translocation. (A) GFP-tagged ßarr2 was expressed in HEK cells without GRK overexpression (Left panel) or co-expresssed with wild-type Lgr5/-GRK or Lgr5/+GRK (Right panel and Middle panel, respectively). (B) GFP-tagged Barr2 was cotransfected with GRK6 and the indicated Lgr5 C-tail mutant. The primary amino acid sequence of mutants are provided in tabular form. (Table 18.1 -12). Native GFP fluorescence was imaged using confocal microscopy at $100 \mathrm{X}$.

doi: 10.1371 /journal.pone.0084476.g008

constitutive internalization of Lgr5. These data suggest that these sites act as priming residues for subsequent phosphorylation at $873-875$ and that the internalization program must be initiated for recruitment of ßarr2. Moreover, they demonstrate that constitutive internalization can be 


\section{Figure 9}

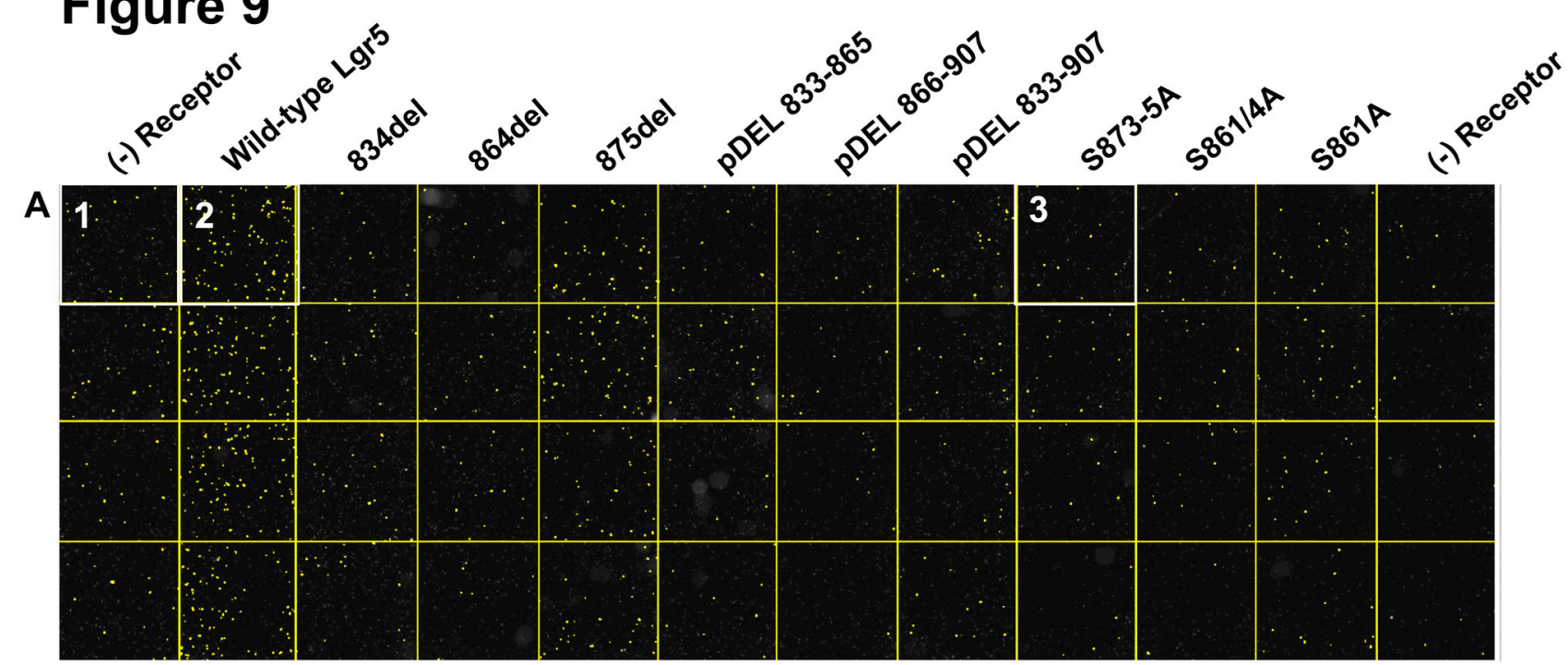

B

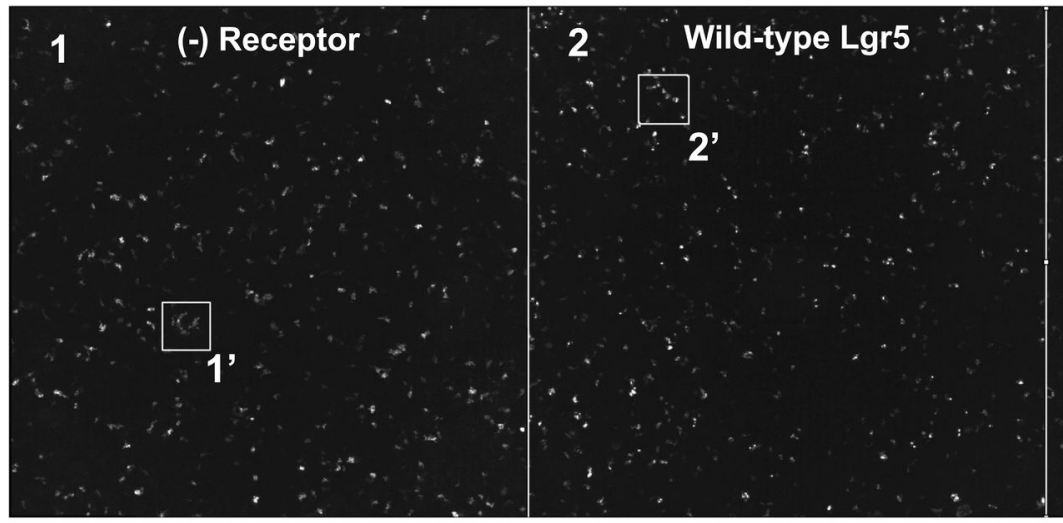

3

S873-5A

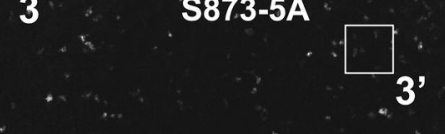

C

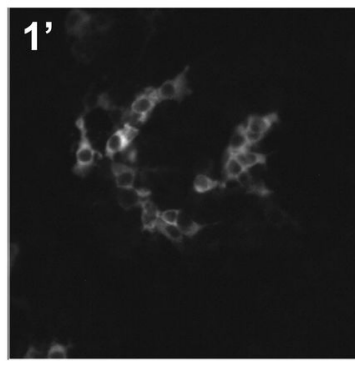

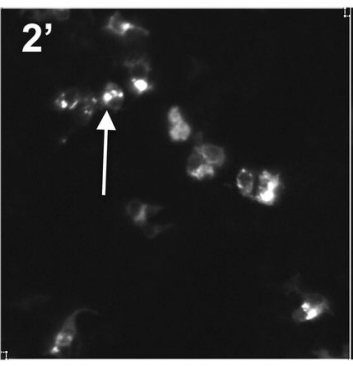

$3^{3}$

D

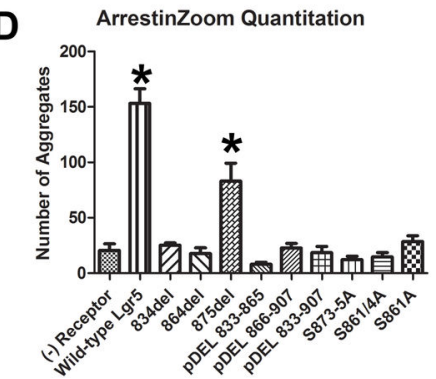

Figure 9. Quantitation of Barr2 recruitment to Lgr5 using an ArrestinZoom Assay (Part 1). GFP-tagged ßarr2 and GRK-6 were transiently co-expressed together with the permutations of Lgr5 previously described for confocal analysis and reviewed in Table 1. (A) 48-wells of a 96-well plate were imaged for GFP fluorescence. For each well, 32 images were tiled at $108 \mathrm{X}$ magnification and subsequently stitched together. Stitched images were imported into ImageJ and converted to a single montage where each column represents a different experimental condition (as labeled) and each row designates a technical replicate (3.34X Magnification). As described in materials and methods, Barr2-GFP aggregates were identified (highlighted in yellow). (B) Areas outlined in (A) for (1) Barr2-GFP + GRK-6, (2) Barr2-GFP + GRK-6 + Wild-type Lgr5, and (3) Barr2-GFP + GRK-6 + S873-5A at 8.9X magnification (C) Areas outlined in $\mathrm{B}$, presented at 108X magnification (Arrow in 2' denotes ßarr2 aggregates, that are absent without Lgr5 and when the "SSS" cluster is mutated to "AAA"). (D) Highlighted Barr2 aggregates in "A" were quantitated, graphed, and tested for statistically significant differences by 1-way ANOVA and post hoc Bonferroni correction for multiple comparisons $(p<0.05)$. The results are representative of three independent experiments.

doi: 10.1371/journal.pone.0084476.g009 


\section{Figure 10}
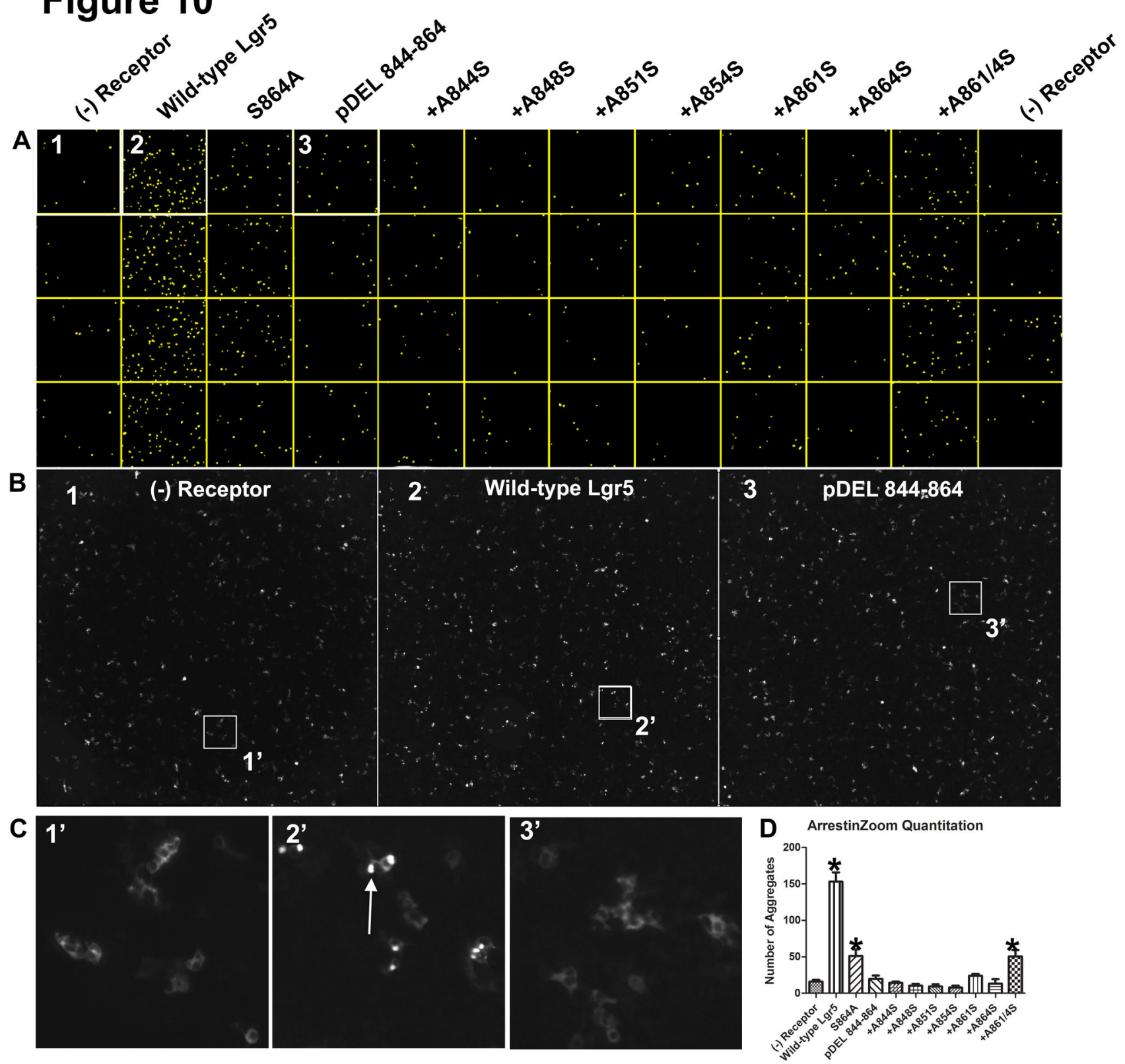

Figure 10. Quantitation of Barr2 recruitment to Lgr5 using an ArrestinZoom Assay (Part 2). GFP-tagged ßarr2 and GRK-6 were transiently co-expressed together with the permutations of Lgr5 previously described for confocal analysis and reviewed in Table 1. (A) 48-wells of a 96-well plate were imaged for GFP fluorescence. For each well, 32 images were tiled at $108 \mathrm{X}$ magnification and subsequently stitched together. Stitched images were imported into ImageJ and converted to a single montage where each column represents a different experimental condition (as labeled) and each row designates a technical replicate (3.34X Magnification). As described in materials and methods, Barr2-GFP aggregates were identified and are shown in yellow. (B) Areas outlined in (A) for (1) Barr2-GFP + GRK-6, (2) Barr2-GFP + GRK-6 + Wild-type Lgr5, and (3) Barr2-GFP + GRK-6 + pDEL 844-864 at $8.9 \mathrm{X}$ magnification $(\mathrm{C})$ Areas outlined in $\mathrm{B}$, presented at 108X magnification (Arrow in 2' denotes ßarr2 aggregates, that are absent without Lgr5 and when the phosphor-acceptors between amino acids 844-864 are mutated to alanine; pDEL844-864). (D) Highlighted Barr2 aggregates in "A" were quantitated, graphed, and tested for statistically significant differences by 1-way ANOVA and post hoc Bonferroni correction for multiple comparisons $(p<0.05)$. The results are representative of three independent experiments.

doi: 10.1371/journal.pone.0084476.g010 
effectively segregated from ßarr2-coupling in the absence of a ligand.

We previously concluded that based upon its primary amino acid sequence that either "the potent $\beta$-arrestin binding domain in the Lgr5 tail is a vestigial motif, or more provocatively, it could indicate the existence of another class of endogenous Lgr5 ligands [24]." Our data provide the first experimental evidence that the "SSS" cluster is more than a vestigial motif and that under the appropriate conditions is a functional ßarr2 high-affinity motif supportive of typical GPCR behaviors.

The GRK overexpression studies presented herein provide interesting insight into the molecular details of this process. Seven mammalian GRKs exist and are classified into 3 groups that include GRK1-like, GRK2-like, and GRK4-like corresponding to GRK1/7, GRK2/3, and GRK4/5/6 respectively $[47,48]$. The GRK1-like subfamily is expressed in the retina and therefore we only focused on the GRK2- and GRK4-like subfamily. Our studies demonstrated that overexpression of GRK4-like but not GRK2-like family members are able to stimulate recruitment of $\beta$ arr2 to WT Lgr5. The ability to promote ligand-independent arrestin translocation has been shown previously for a mutant plasma membrane localized and $G$ protein independent form of GRK2 [49]. In contrast, our studies use WT versions of GRKs to demonstrate the agonistindependent translocation of ßarr2 to a receptor. The overexpression of GRK4 and GRK6 has also been shown to phosphorylate and cause endocytosis of the $\beta 2 A R$ in the absence of ligand [46]. Previous in silico findings suggested that the serine cluster "SSS" (aa873-875) in Lgr5 is a likely candidate for GRK phosphorylation [24]. These data, coupled to reports demonstrating that FSHR is tightly regulated by the repertoire of GRKs expressed, further suggest that overexpression of GRK4-like family members phosphorylate Lgr5 and stimulate ßarr2 recruitment $[5,50]$.

The disparate activity of GRK2-like and GRK4-like family kinases found in this study further validate previous reports proposing a GRK mediated phosphorylation barcode of GPCRs. Interestingly, while GRK2 and GRK4 family kinases can phosphorylate the same receptor, it has been demonstrated for a few GPCRs that GRK2-like kinases might phosphorylate residues responsible for the desensitization process whereas GRK4-like kinases phosphorylate residues involved in mediating $G$ protein-independent or arrestin mediated signaling [51]. Together with our previous report outlining the molecular determinants of Lgr5 internalization [24], these findings raise the possibility that serines $861 / 864$ might be GRK2-like kinase substrates which mediate endocytosis while the "SSS" (873-875) are the substrates of GRK4-like kinases and are essential for high-affinity ßarr2 coupling and downstream signaling. These important concepts will be the subject of future investigations.

The fact that the known high-affinity ligands to Lgr4-6 are unable to stimulate $\beta$ arr2 recruitment or $G$ protein-coupling is a paradoxical finding given the homology between Lgr4-6 and Lgr1-3 and the ability of Lgr1-3 ligands to elicit typical GPCR behaviors. The GRK overexpression experiments presented in this study suggest Lgr5 is able to support the ßarr2 component of typical GPCR behaviors. Interestingly, a handful of other
non-GPCRs have shown to be either substrates of GRKs or able to bind arrestins. However, the GPCR class of receptors differ strikingly in their structural characteristics and protein motifs [52,53]. Importantly, Lgr5 possesses the defining structural characteristics and protein motifs inherent to GPCRs and at the appropriate conserved structural locations. In addition to the 7-TM bundle, Lgr5 contains canonical "DRY", "NPXXY", and "SSS" serine cluster motifs (Figure 1 and Figure 2). Moreover, during review of our manuscript, a single report was published indicating that Lgr5 may couple to the heterotrimeric $G$ protein alpha subunits $G_{12 / 13}$ [54]. Therefore, these recent data, together with the multiple lines of structural, biochemical, and cell biological evidence that we present here, indicate that Lgr5 possesses the capacity for classical GPCR behavior. Collectively, these results illustrate that reclassification of Lgr5 as a non-GPCR is premature, and point to a significant knowledge gap in our understanding of Lgr4-6 signaling.

The high-affinity interactions described for R-spondins or Norrin with Lgr4-6 are indisputable, raising the question of what the roles are for each of these ligands in Lgr4- 6 biology? The ability to transduce Wnt signaling is intricately regulated by the internalization of Lgr5 and Lgr4 [15,55]. As discussed earlier, we have previously shown that internalization of Lgr5 likely occurs independently of the ßarr2 recruitment motif [24]. Therefore, it is altogether likely that Rspondin signaling through Lgr4-6 highlights a unique signaling role that may occur independently of a more typical GPCR signal transduction paradigm. Interestingly, a high-resolution structure of Rspondin-1 with the Lgr4 ectodomain support the notion that Rspondins don't engage the N-terminus of Lgr4 in a manner conducive to $G$ protein coupling, despite the existence of the necessary structural domains within the receptor [56]. These findings continue to demonstrate the many fascinating aspects of GPCRs including their ability to couple to multiple signaling pathways independent of G proteins or arrestins but shouldn't discount the existence of alternative ligands or effectors capable of their activation.

The discovery of Norrin as a high-affinity ligand to Lgr4-6 was guided by an evolutionary search for mammalian orthologues of the Drosophila bursicon, a heterodimer of burs/ pburs and the ligand for the Drosophila Lgr4 orthologue, Lgr2. This analysis revealed that Norrin is closely related to burs/ pburs as revealed by its cysteine-knot motif comprised of 11 cysteine residues [17,57]. Like mammalian Lgr1-3 ligands, Drosophila bursicon is only active as a complete heterodimer [57-59]. In contrast Norrin binding and its ability to stimulate Wnt-signaling is independent of heterodimer formation possibly due to its ability to oligomerize [17]. Interestingly, despite binding to Lgr5 and Lgr6, Norrin doesn't stimulate Wntsignaling at these receptors. In contrast to Rspondins, Norrin possess a remarkable evolutionary conservation to bursicon [17]. Despite this similarity, the inability to engage $G$ protein or arrestin signaling with Norrin indicates that a component of this pathway is not well understood.

The C-tail alignments demonstrated that Lgr4 and Lgr6 both lack the "SSS" cluster necessary for high-affinity ßarr2 recruitment (Figure 2). As expected, we were unable to 
demonstrate that Lgr4 recruits $\beta$ arr2 in our ligand-independent GRK assay. The C-tail of Lgr4 is more similar to $\beta 2 A R$ than $V 2 R$. The $\beta 2 A R$ also doesn't support translocation of $\beta$ arr2 in our ligand-independent $\beta$ arr2 assay, presumably since its affinity for ßarr2 is too weak. Therefore, using this assay we can't determine the affinity of Barr2 for Lgr4. For Lgr6, despite much work we are still unable to properly express Lgr6 on the plasma membrane in heterologous cell systems. Instead, we find that Lgr6 continues to be aggregated in the endoplasmic reticulum (ER) (data not shown) indicating that studies investigating Lgr6 signaling need to be carefully evaluated and interpreted. Moreover, Lgr6 completely lacks the "DRY" motif in IC2 suggesting that signaling at Lgr6 may diverge significantly from Lgr4 and Lgr5.

In the course of these studies we developed the ArrestinZoom assay to quickly, reliably, and quantitatively assess ßarr2 recruitment to Lgr5 and its mutants. There are currently several techniques in place to quantify Barrestin recruitment in cell assays that for the small laboratory include cost-prohibitive high-content imaging systems, or one dimensional optical readers of bioluminescence energy transfer, enzyme complementation, or transcriptional reporter assays $[43,44]$. These systems, while effective, can be either expensive or require the generation of assay specific receptor constructs utilizing C-tail receptor/reporter fusions that may alter arrestin affinity. In our analysis, we found the ArrestinZoom assay was able to reliably quantify arrestin recruitment for the majority of constructs tested. The minor discrepancies between it and confocal imaging can probably be resolved using alternative computer algorithms for image processing and utilizing higher resolution cameras. ßarr2-GFP translocation to activated GPCRs is a powerful screening tool for the small laboratory. As with other similar methodologies, the potential remains for loss of Arrestin/Receptor affinity due to fusion proteins and additional requirements for validating assays since compounds may conceivably activate ßarrestin translocation independent of receptor activation [60]. Even so, the findings with the ArrestinZoom assay, demonstrating that Barr2 recruitment to Lgr5 can be quantified by moderate throughput using high content imaging at relatively low cost, indicate that this approach can significantly enhance the ability of small laboratories to study receptor and protein trafficking.

The potential to directly regulate stem cell activity on the basis of Lgr-targeted therapy is of incredible interest. Unfortunately, the inability to identify endogenous ligands and effectors of Lgr4-6 that support GPCR signaling continues to slow their full biochemical characterization and the search for novel drug therapy. The results of our study demonstrate that Lgr5 possesses the capacity for typical GPCR behaviors. This study is an important first-step in evaluating roles for Lgr4-6 as GPCRs, and provides the rationale for future work, which will explore the missing signaling effectors capable of eliciting these behaviors.

\section{Supporting Information}

Figure S1. GRK overexpression is required for $\beta$ arr2 translocation to Lgr5. (A) GRK5 and (B) GRK6 antibody staining. As described in materials and methdods, HEK293T cells were plated and transfected with 3xHA-Lgr5 or 3xHA-834del Lgr5, ßarr2-GFP, and GRK5 or GRK6, as indicated at left. Cells were fixed, permeabilized, stained, and imaged for the HA epitope (Blue, 633nm secondary dectection), GRK5 or GRK6 (Red, 568nm secondary detection), Barr2-GFP (Green, Native fluoresence). A merge of all three channels is presented at the right. Endogenous GRK5 or GRK6 are below the levels of antibody detection and are unable to promote ligand-independent ßarr2-GFP translocation. Overexpression of GRK5 or GRK6 enabled translocation of Barr2 to Lgr5 that was depedent upon the Lgr5 C-tail. (TIF)

Figure S2. Lgr5 does not recruit ßarr1 in the ligandindependent translocation assay. HEK293T cells wereplated on 35mm glass-bottom dishes (MatTek: P35G-0-10C) and transfected with wild-type human V2R or Lgr5 $(2 \mu \mathrm{g})$, Barr1GFP $(1.5 \mu \mathrm{g})$, and [+/- $(2 \mu \mathrm{g})]$ GRK2, GRK4, GRK5, or GRK6 as indicated. Cells were imaged live at $100 \mathrm{X}$ by confocal microscopy (ßarr1-GFP, Green). (A) In a control experiment, V2R and Barr1-GFP transfected cells were imaged live before and 25 minutes after $10 \mu \mathrm{M}$ vasopressin stimulus (AVP: Sigma V9879). As expected, AVP stimulus of V2R caused translocation of $\beta$ arr1-GFP to vesicles.(B) In contrast to the ligand-independent GRK assay described in the manuscript, GRK overexpression alone is unable to promote ßarr1-GFP translocation for the V2R or Lgr5.

(TIF)

\section{File S1. Class 1 GPCR Alignment.} (TXT)

\section{Acknowledgements}

We thank Sean Peterson and Tom Pack for their thoughtful review of the manuscript. We also thank Tom Pack, Gabor Turu, and P.J. Nicholls for ImageJ advice. We are also grateful for the GRK antibodies that were kind gifts of Dr. Richard Premont.

\section{Author Contributions}

Conceived and designed the experiments: JCS LKR LSB MGC. Performed the experiments: JCS LKR. Analyzed the data: JCS LKR. Contributed reagents/materials/analysis tools: JCS LKR LSB MGC. Wrote the manuscript: JCS LSB MGC. 


\section{References}

1. Hsu SY, Liang S-G, Hsueh AJW (1998) Characterization of Two LGR Genes Homologous to Gonadotropin and Thyrotropin Receptors with Extracellular Leucine-Rich Repeats and a G Protein-Coupled, SevenTransmembrane. Region - Molecular Endocrinology 12: 1830-1845. doi:10.1210/me.12.12.1830.

2. McDonald T, Wang R, Bailey W, Xie G, Chen F et al. (1998) Identification and cloning of an orphan $G$ protein-coupled receptor of the glycoprotein hormone receptor subfamily. Biochem Biophys Res Commun 247: 266-270. doi:10.1006/bbrc.1998.8774. PubMed: 9642114.

3. Hsu SY, Kudo M, Chen T, Nakabayashi K, Bhalla A et al. (2000) The three subfamilies of leucine-rich repeat-containing $G$ protein-coupled receptors (LGR): identification of LGR6 and LGR7 and the signaling mechanism for LGR7. Mol Endocrinol 14: 1257-1271. doi:10.1210/me. 14.8.1257. PubMed: 10935549.

4. Schubert RL, Narayan P, Puett D (2003) Specificity of cognate ligandreceptor interactions: fusion proteins of human chorionic gonadotropin and the heptahelical receptors for human luteinizing hormone, thyroidstimulating hormone, and follicle-stimulating hormone. Endocrinology 144: 129-137. doi:10.1210/en.2002-220829. PubMed: 12488338.

5. Kara E, Crépieux P, Gauthier C, Martinat N, Piketty V et al. (2006) A phosphorylation cluster of five serine and threonine residues in the $\mathrm{C}$ terminus of the follicle-stimulating hormone receptor is important for desensitization but not for beta-arrestin-mediated ERK activation. Mol Endocrinol 20: 3014-3026. doi:10.1210/me.2006-0098. PubMed: 16887887.

6. Nakamura K, Liu X, Ascoli M (2000) Seven non-contiguous intracellular residues of the lutropin/choriogonadotropin receptor dictate the rate of agonist-induced internalization and its sensitivity to non-visual arrestins. J Biol Chem 275: 241-247. doi:10.1074/jbc.275.1.241. PubMed: 10617611.

7. Frenzel R, Voigt C, Paschke R (2006) The human thyrotropin receptor is predominantly internalized by beta-arrestin 2. Endocrinology 147: 3114-3122. doi:10.1210/en.2005-0687. PubMed: 16513835.

8. Barker N, van Es JH, Kuipers J, Kujala P, van den Born M et al. (2007) Identification of stem cells in small intestine and colon by marker gene Lgr5. Nature 449: 1003-1007. doi:10.1038/nature06196. PubMed: 17934449.

9. Barker N, Tan S, Clevers H (2013) Lgr proteins in epithelial stem cell biology. Development 140: 2484-2494. doi:10.1242/dev.083113. PubMed: 23715542.

10. Leushacke M, Barker N (2012) Lgr5 and Lgr6 as markers to study adult stem cell roles in self-renewal and cancer. Oncogene 31: 3009-3022. doi:10.1038/onc.2011.479. PubMed: 22002312.

11. Kemper K, Prasetyanti PR, De Lau W, Rodermond H, Clevers $\mathrm{H}$ et al. (2012) Monoclonal antibodies against Lgr5 identify human colorectal cancer stem cells. Stem Cells 30: 2378-2386. doi:10.1002/stem.1233. PubMed: 22969042.

12. de Lau W, Barker N, Low TY, Koo BK, Li VS et al. (2011) Lgr5 homologues associate with Wnt receptors and mediate R-spondin signalling. Nature 476: 293-297. doi:10.1038/nature10337. PubMed: 21727895 .

13. Carmon KS, Gong X, Lin Q, Thomas A, Liu Q (2011) R-spondins function as ligands of the orphan receptors LGR4 and LGR5 to regulate Wnt/beta-catenin signaling. Proc Natl Acad Sci U S A 108: 11452-11457. doi:10.1073/pnas.1106083108. PubMed: 21693646.

14. Glinka A, Dolde C, Kirsch N, Huang YL, Kazanskaya O et al. (2011) LGR4 and LGR5 are R-spondin receptors mediating Wnt/beta-catenin and Wnt/PCP signalling. EMBO Rep 12: 1055-1061. doi:10.1038/ embor.2011.175. PubMed: 21909076.

15. Carmon KS, Lin Q, Gong X, Thomas A, Liu Q (2012) LGR5 Interacts and Cointernalizes with Wnt Receptors To Modulate Wnt/beta-Catenin Signaling. Mol Cell Biol 32: 2054-2064. doi:10.1128/MCB.00272-12. PubMed: 22473993.

16. Gong X, Carmon KS, Lin Q, Thomas A, Yi J et al. (2012) LGR6 Is a High Affinity Receptor of R-Spondins and Potentially Functions as a Tumor Suppressor. PLOS ONE 7: e37137. doi:10.1371/journal.pone. 0037137. PubMed: 22615920

17. Deng C, Reddy P, Cheng Y, Luo CW, Hsiao CL et al. (2013) Multifunctional norrin is a ligand for the LGR4 receptor. J Cell Sci 126: 2060-2068. doi:10.1242/jcs.123471. PubMed: 23444378

18. Gainetdinov RR, Premont RT, Bohn LM, Lefkowitz RJ, Caron MG (2004) DESENSITIZATION OF G PROTEIN-COUPLED RECEPTORS AND NEURONAL FUNCTIONS. Annu Rev Neurosci 27: 107-144. doi: 10.1146/annurev.neuro.27.070203.144206. PubMed: 15217328
19. Rajagopal S, Rajagopal K, Lefkowitz RJ (2010) Teaching old receptors new tricks: biasing seven-transmembrane receptors. Nat Rev Drug Discov 9: 373-386. doi:10.1038/nrd3024. PubMed: 20431569.

20. Ferguson SS, Downey WE 3rd, Colapietro AM, Barak LS, Ménard L et al. (1996) Role of beta-arrestin in mediating agonist-promoted G protein-coupled receptor internalization. Science 271: 363-366. doi: 10.1126/science.271.5247.363. PubMed: 8553074.

21. Beaulieu JM, Gainetdinov RR, Caron MG (2009) Akt/GSK3 signaling in the action of psychotropic drugs. Annu Rev Pharmacol Toxicol 49: 327-347. doi:10.1146/annurev.pharmtox.011008.145634. PubMed: 18928402.

22. Laporte SA, Oakley RH, Holt JA, Barak LS, Caron MG (2000) The interaction of beta-arrestin with the AP-2 adaptor is required for the clustering of beta 2-adrenergic receptor into clathrin-coated pits. J Biol Chem 275: 23120-23126. doi:10.1074/jbc.M002581200. PubMed: 10770944.

23. Laporte SA, Oakley RH, Zhang J, Holt JA, Ferguson SS et al. (1999) The beta2-adrenergic receptor/betaarrestin complex recruits the clathrin adaptor AP-2 during endocytosis. Proc Natl Acad Sci U S A 96: 3712-3717. doi:10.1073/pnas.96.7.3712. PubMed: 10097102.

24. Snyder JC, Rochelle LK, Lyerly HK, Caron MG, Barak LS (2013) Constitutive Internalization of the Leucine-rich $G$ protein-coupled Receptor-5 (LGR5) to the trans-Golgi. Network. J - Biol Chem.

25. Kapur A, Zhao P, Sharir H, Bai Y, Caron MG et al. (2009) Atypical responsiveness of the orphan receptor GPR55 to cannabinoid ligands. J Biol Chem 284: 29817-29827. doi:10.1074/jbc.M109.050187. PubMed: 19723626.

26. Zhang J, Barak LS, Anborgh PH, Laporte SA, Caron MG et al. (1999) Cellular trafficking of $\mathrm{G}$ protein-coupled receptor/beta-arrestin endocytic complexes. J Biol Chem 274: 10999-11006. doi:10.1074/jbc. 274.16.10999. PubMed: 10196181.

27. Sharman JL, Benson HE, Pawson AJ, Lukito V, Mpamhanga CP et al. (2013) IUPHAR-DB: updated database content and new features. Nucleic Acids Res 41: D1083-D1088. doi:10.1093/nar/gks960. PubMed: 23087376.

28. Sievers F, Wilm A, Dineen D, Gibson TJ, Karplus K et al. (2011) Fast, scalable generation of high-quality protein multiple sequence alignments using Clustal. Omega - Mol Syst Biol 7: 539.

29. Goujon M, McWilliam H, Li W, Valentin F, Squizzato S et al. (2010) A new bioinformatics analysis tools framework at EMBL-EBI. Nucleic Acids Res 38: W695-W699. doi:10.1093/nar/gkq313. PubMed: 20439314.

30. Waterhouse AM, Procter JB, Martin DM, Clamp M, Barton GJ (2009) Jalview Version 2--a multiple sequence alignment editor and analysis workbench. Bioinformatics 25: 1189-1191

31. Kingston RE, Chen CA, Okayama H (2001) Calcium Phosphate Transfection. Current Protocols in Immunology. John Wiley \& Sons, Inc.

32. Schneider CA, Rasband WS, Eliceiri KW (2012) NIH Image to ImageJ: 25 years of image analysis. Nat Methods 9: 671-675. doi:10.1038/ nmeth.2089. PubMed: 22930834.

33. Barak LS, Tiberi M, Freedman NJ, Kwatra MM, Lefkowitz RJ et al. (1994) A highly conserved tyrosine residue in G protein-coupled receptors is required for agonist-mediated beta 2-adrenergic receptor sequestration. J Biol Chem 269: 2790-2795. PubMed: 7507928.

34. Barak LS, Ménard L, Ferguson SS, Colapietro AM, Caron MG (1995) The conserved seven-transmembrane sequence NP(X)2,3Y of the Gprotein-coupled receptor superfamily regulates multiple properties of the beta 2-adrenergic receptor. Biochemistry 34: 15407-15414. doi: 10.1021/bi00047a003. PubMed: 7492540.

35. Marion S, Oakley RH, Kim KM, Caron MG, Barak LS (2006) A betaarrestin binding determinant common to the second intracellular loops of rhodopsin family $G$ protein-coupled receptors. J Biol Chem 281: 2932-2938. PubMed: 16319069

36. Barak LS, Oakley RH, Laporte SA, Caron MG (2001) Constitutive arrestin-mediated desensitization of a human vasopressin receptor mutant associated with nephrogenic diabetes insipidus. Proc Natl Acad Sci U S A 98: 93-98. doi:10.1073/pnas.98.1.93. PubMed: 11134505

37. Wilbanks AM, Laporte SA, Bohn LM, Barak LS, Caron MG (2002) Apparent Loss-of-Function Mutant GPCRs Revealed as Constitutively Desensitized Receptors†. Biochemistry 41: 11981-11989. doi:10.1021/ bi020275m. PubMed: 12356298.

38. Oakley RH, Laporte SA, Holt JA, Caron MG, Barak LS (2000) Differential affinities of visual arrestin, beta arrestin1, and beta arrestin2 for $G$ protein-coupled receptors delineate two major classes of receptors. J Biol Chem 275: 17201-17210. doi:10.1074/ jbc.M910348199. PubMed: 10748214. 
39. Oakley RH, Laporte SA, Holt JA, Barak LS, Caron MG (2001) Molecular determinants underlying the formation of stable intracellular $\mathrm{G}$ protein-coupled receptor-beta-arrestin complexes after receptor endocytosis*. J Biol Chem 276: 19452-19460. doi:10.1074/ jbc.M101450200. PubMed: 11279203.

40. Oakley RH, Laporte SA, Holt JA, Barak LS, Caron MG (1999) Association of beta-arrestin with $G$ protein-coupled receptors during clathrin-mediated endocytosis dictates the profile of receptor resensitization. J Biol Chem 274: 32248-32257. doi:10.1074/jbc. 274.45.32248. PubMed: 10542263.

41. Salon JA, Lodowski DT, Palczewski K (2011) The significance of G protein-coupled receptor crystallography for drug discovery. Pharmacol Rev 63: 901-937. doi:10.1124/pr.110.003350. PubMed: 21969326.

42. Barak LS, Ferguson SS, Zhang J, Caron MG (1997) A beta-arrestin/ green fluorescent protein biosensor for detecting $G$ protein-coupled receptor activation. J Biol Chem 272: 27497-27500. doi:10.1074/jbc. 272.44.27497. PubMed: 9346876

43. Verkaar F, van Rosmalen JW, Blomenröhr M, van Koppen CJ, Blankesteijn WM et al. (2008) G protein-independent cell-based assays for drug discovery on seven-transmembrane receptors. Biotechnol Annu Rev 14: 253-274. doi:10.1016/S1387-2656(08)00010-0. PubMed: 18606367.

44. Zhang $R$, Xie $X$ (2012) Tools for GPCR drug discovery. Acta Pharmacol Sin 33: 372-384. doi:10.1038/aps.2011.173. PubMed: 22266728.

45. Ferguson SS, Ménard L, Barak LS, Koch WJ, Colapietro AM et al. (1995) Role of phosphorylation in agonist-promoted beta 2-adrenergic receptor sequestration. Rescue of a sequestration-defective mutant receptor by beta ARK1. J Biol Chem 270: 24782-24789. doi:10.1074/ jbc. 270.42.24782. PubMed: 7559596.

46. Ménard L, Ferguson SS, Barak LS, Bertrand L, Premont RT et al. (1996) Members of the $G$ protein-coupled receptor kinase family that phosphorylate the beta2-adrenergic receptor facilitate sequestration. Biochemistry 35: 4155-4160. doi:10.1021/bi952961+ PubMed: 8672451

47. Evron T, Daigle TL, Caron MG (2012) GRK2: multiple roles beyond G protein-coupled receptor desensitization. Trends Pharmacol Sci 33: 154-164. doi:10.1016/j.tips.2011.12.003. PubMed: 22277298.

48. Premont RT, Gainetdinov RR (2007) Physiological roles of G proteincoupled receptor kinases and arrestins. Annu Rev Physiol 69: 511-534. doi:10.1146/annurev.physiol.69.022405.154731. PubMed: 17305472.

49. Oakley RH, Hudson CC, Sjaastad MD, Loomis CR (2006) The ligandindependent translocation assay: an enabling technology for screening orphan $\mathrm{G}$ protein-coupled receptors by arrestin recruitment. Methods
Enzymol 414: 50-63. doi:10.1016/S0076-6879(06)14004-5. PubMed: 17110186.

50. Troispoux C, Guillou F, Elalouf JM, Firsov D, lacovelli L et al. (1999) Involvement of $G$ protein-coupled receptor kinases and arrestins in desensitization to follicle-stimulating hormone action. Mol Endocrinol 13: 1599-1614. doi:10.1210/me.13.9.1599. PubMed: 10478849.

51. Nobles KN, Xiao K, Ahn S, Shukla AK, Lam CM et al. (2011) Distinct phosphorylation sites on the beta(2)-adrenergic receptor establish a barcode that encodes differential functions of beta-arrestin. Sci Signal 4: ra51. PubMed: 21868357.

52. Gurevich VV, Gurevich EV (2006) The structural basis of arrestinmediated regulation of G-protein-coupled receptors. Pharmacol Ther 110: 465-502. doi:10.1016/j.pharmthera.2005.09.008. PubMed: 16460808.

53. Gurevich EV, Tesmer JJ, Mushegian A, Gurevich VV (2012) G proteincoupled receptor kinases: more than just kinases and not only for GPCRs. Pharmacol Ther 133: 40-69. doi:10.1016/j.pharmthera. 2011.08.001. PubMed: 21903131

54. Kwon MS, Park BO, Kim HM, Kim S (2013) Leucine-rich repeatcontaining G-protein coupled receptor 5/GPR49 activates G12/13-Rho GTPase pathway. Mol Cells 36: 267-272. doi:10.1007/ s10059-013-0173-z. PubMed: 23912594.

55. Hao HX, Xie Y, Zhang Y, Charlat O, Oster E et al. (2012) ZNRF3 promotes Wnt receptor turnover in an R-spondin-sensitive manner. Nature 485: 195-200. doi:10.1038/nature11019. PubMed: 22575959.

56. Wang D, Huang B, Zhang S, Yu X, Wu W et al. (2013) Structural basis for R-spondin recognition by LGR4/5/6 receptors. Genes Dev 27: 1339-1344. doi:10.1101/gad.219360.113. PubMed: 23756652

57. Luo CW, Dewey EM, Sudo S, Ewer J, Hsu SY et al. (2005) Bursicon, the insect cuticle-hardening hormone, is a heterodimeric cystine knot protein that activates $\mathrm{G}$ protein-coupled receptor LGR2. Proc Natl Acad Sci U S A 102: 2820-2825. doi:10.1073/pnas.0409916102. PubMed: 15703293.

58. Harwood BN, Fortin JP, Gao K, Chen C, Beinborn M et al. (2013) Membrane tethered bursicon constructs as heterodimeric modulators of the Drosophila G protein-coupled receptor rickets. Mol Pharmacol 83: 814-821. doi:10.1124/mol.112.081570. PubMed: 23340494.

59. Hearn MT, Gomme PT (2000) Molecular architecture and biorecognition processes of the cystine knot protein superfamily: part I. The glycoprotein hormones. J Mol Recognit 13: 223-278. doi: 10.1002/1099-1352(200009/10)13:5. PubMed: 10992290.

60. Barak LS, Bai Y, Snyder JC, Wang J, Chen W et al. (2013) Triphenylmethane Dye Activation of Beta-Arrestin. Biochemistry, 52: 5403-14. PubMed: 23865508. 